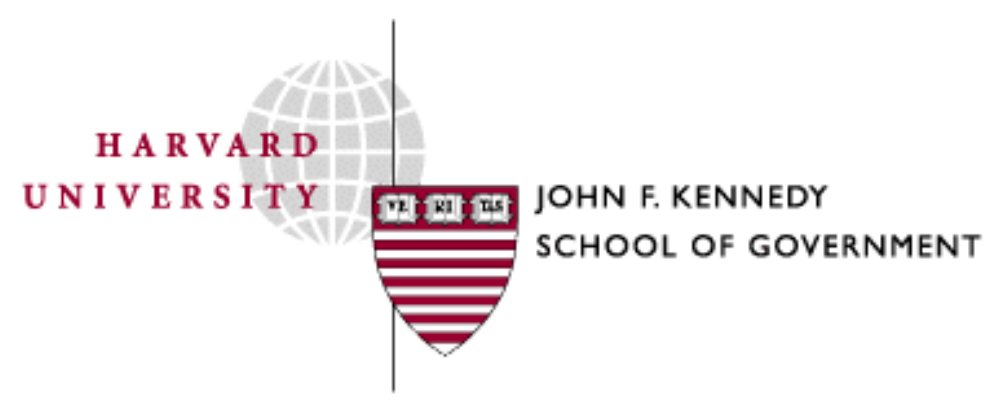

Faculty Research Working Papers Series

\title{
Polynomial Approximations and Supply Function Equilibrium Stability (Aug-04)
}

\author{
Ross Baldick and William W. Hogan
}

March 2005

RWP05-028

The views expressed in the KSG Faculty Research Working Paper Series are those of the author(s) and do not necessarily reflect those of the John F. Kennedy School of Government or Harvard University. Copyright belongs to the author(s). Papers may be downloaded for personal use only. 


\title{
POLYNOMIAL APPROXIMATIONS AND
}

\section{SUPPLY FUNCTION EQUILIBRIUM STABILITY}

\author{
Ross Baldick \\ University of Texas \\ William W. Hogan \\ Harvard University
}

August 27, 2004

Center for Business and Government

John F. Kennedy School of Government

Harvard University

Cambridge, Massachusetts 02138 


\section{CONTENTS}

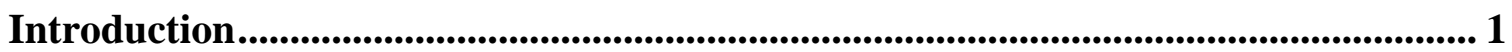

Supply Function Best Response........................................................................... 2

Symmetric Unconstrained SFE Polynomial Approximation ............................................. 7

Regression Approximation Rule.......................................................................................... 10

Taylor Series Approximation Rule................................................................................ 12

Eigenvalue Sensitivity Tests .............................................................................................. 19

Modeling Oligopoly Behavior ............................................................................................ 25

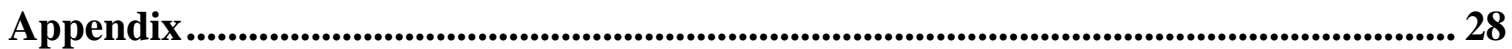

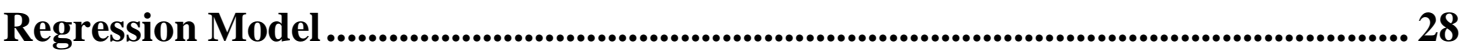

Taylor Approximation Model............................................................................................ 33

Exact SFE Model....................................................................................................................... 40 


\title{
Polynomial Approximations and Supply Function Equilibrium Stability
}

\author{
Ross Baldick $^{\mathrm{i}}$ and William W. Hogan ${ }^{\mathrm{ii}}$
}

August 27, 2004

\begin{abstract}
Organized electricity markets often require submission of supply functions ahead of the realization of uncertain demand. As a model of oligopoly behavior, the Nash condition of supply function equilibrium has a natural appeal. Typically this produces a continuum of possible equilibria, presenting an equilibrium selection problem. Beyond existence, stability of an equilibrium would be an obvious criterion for selection. For affine demand and marginal costs, polynomial approximation provides an approach for analyzing the stability of unconstrained supply function equilibria. The set of stable approximation equilibria is small and its properties suggest that the set of stable exact supply function equilibria is empty.
\end{abstract}

\section{Introduction}

Supply function equilibrium (SFE) provides an appealing framework for modeling strategic interactions among suppliers in electricity markets. ${ }^{1}$ The organized electricity markets often require submission of supply functions ahead of the realization of uncertain demand, thereby conforming to a key assumption of the SFE model. An SFE is a Nash equilibrium in supply functions. However, characterizing a supply function equilibrium is difficult in practice.

If the SFE model is a good predictor of oligopoly behavior, it needs to be well understood for purposes of market power modeling, simulation of market outcomes and monitoring of market performance. However, theoretical research and computational investigations have revealed substantial complexity and persistent numerical difficulties in computing equilibrium solutions without ad hoc limitations on the formulation.

The simplest case is for an unconstrained SFE in a one-shot, single-price, marketclearing setting with affine marginal costs, no capacity constraints and no contracts. ${ }^{2}$ Here we examine this ideal case to consider the existence and stability of equilibria. In the SFE model there could be a continuum of equilibria, presenting the problem of selecting among the possible solutions to characterize the market equilibrium. Given

\footnotetext{
1 Paul D. Klemperer and Margaret A. Meyer. "Supply Function Equilibria in Oligopoly Under Uncertainty,” Econometrica, Vol. 57, No. 6, November 1989, pp. 1243-1277. Richard Green and David M. Newbery, "Competition in the British Electricity Spot Market,” Journal of Political Economy, Vol. 100, No. 5, October 1992, pp. 929-953.

2 The constraints refer to the monotonicity requirements typically imposed on supply function bids. The unconstrained SFEs are monotonic solutions of the optimality conditions as discussed below.
} 
perturbations from a Nash equilibrium in supply functions, the system may be unstable and not converge to the equilibrium. One approach to reduce the equilibrium selection problem would be to rule out any such unstable solutions as being unsustainable in a dynamic market setting.

The SFE stands in contrast to the competitive solution with price-taking firms. In this non-strategic case the profit maximizing supply function is the marginal cost function, and is both unique and stable. Hence, the analysis of stability is of interest in an examination of the exercise of market power but does not arise with price-taking behavior.

Here we show that with less than a perfect estimation of the other firms' supply functions the number of possible equilibrium solutions is greatly reduced. Further, the remaining equilibrium solutions tend to be unstable under approximation. The instability is more pronounced for the least competitive solutions, suggesting a difficulty in maintaining a significant exercise of market power.

In particular, using polynomial approximations of degree $n$, it appears that all equilibrium solutions are unstable for sufficiently large $n$. Hence, the more flexible the approximation the more likely the approximation equilibrium is unstable. This is shown analytically for the special case of the affine SFE, and supported by numerical investigations with nonlinear SFEs. This suggests a conjecture that the exact unconstrained SFE is chaotic in this sense, with many exact equilibrium solutions but no stable equilibrium.

\section{Supply Function Best Response}

Consider a single market with two firms that submit supply functions before realization of uncertain demand of price-taking buyers. Suppose we have supply functions $S_{i}, S_{j}$ and different quadratic costs $\left(C_{i}(q)=a_{i} q+\frac{1}{2} c_{i} q_{i}^{2}\right)$, affine demand as a function of price $(d=\varphi-\gamma p+\varepsilon)$ and a market setting that determines a single marketclearing price equilibrium after revelation of the demand uncertainty in the additive shock $\varepsilon$. Given the supply function $S_{j}$ of firm $j$, the residual demand of firm $i$ is

$$
\varphi-\gamma p+\varepsilon-S_{j}(p)
$$

Firm $i$ must select its supply to achieve market clearing for each price associated with the realization of the demand shock.

$$
\hat{S}_{i}(p)=\varphi-\gamma p+\varepsilon-S_{j}(p)
$$

In selecting its supply function, firm $i$ would optimize its profit function given as

$$
\pi_{i}(p)=\left(\varphi-\gamma p+\varepsilon-S_{j}(p)\right) p-C_{i}\left(\varphi-\gamma p+\varepsilon-S_{j}(p)\right) .
$$

Let $S_{j}^{(1)}(p)$ denote the $k$ th derivative of $S_{j}(p)$ with respect to $p$. With continuously differentiable $S_{j}$ and quadratic costs, the first-order necessary conditions for optimal profit to determine the price given each realization of the demand shock include 


$$
\hat{S}_{i}(p)=\left(p-C_{i}^{(1)}\left(\hat{S}_{i}(p)\right)\right)\left(\gamma+S_{j}^{(1)}(p)\right)=\left(p-a_{i}-c_{i} \hat{S}_{i}(p)\right)\left(\gamma+S_{j}^{(1)}(p)\right) .
$$

Rearranging terms, this yields the best response $\left(\hat{S}_{i}(p)\right)$ :

$$
\hat{S}_{i}(p)=\frac{\gamma+S_{j}^{(1)}(p)}{1+c_{i}\left(\gamma+S_{j}^{(1)}(p)\right)}\left(p-a_{i}\right) .
$$

We limit attention here to pure strategy equilibria in this continuously differentiable case with emphasis on a bounded interval for demand shocks and the resulting equilibrium price. ${ }^{3}$ Given an unconstrained solution, the SFE must be a fixed point of the system of best response functions

$$
\begin{aligned}
& S_{i}(p)=\frac{\gamma+S_{j}^{(1)}(p)}{1+c_{i}\left(\gamma+S_{j}^{(1)}(p)\right)}\left(p-a_{i}\right), \\
& S_{j}(p)=\frac{\gamma+S_{i}^{(1)}(p)}{1+c_{j}\left(\gamma+S_{i}^{(1)}(p)\right)}\left(p-a_{j}\right) .
\end{aligned}
$$

The unconstrained supply function equilibria depend on the range of possible demand but not on the form of the non-degenerate distribution of the demand shocks. In this sense, the unconstrained SFE is distribution free. When the support of the random demand shock is bounded, it is well known that there may be many SFE solutions, as shown in the following figure.

\footnotetext{
3 Particular market rules may affect the form of the supply functions to include other than
} differentiable functions. 


\section{Continuum of \\ Supply Function Equilibria}

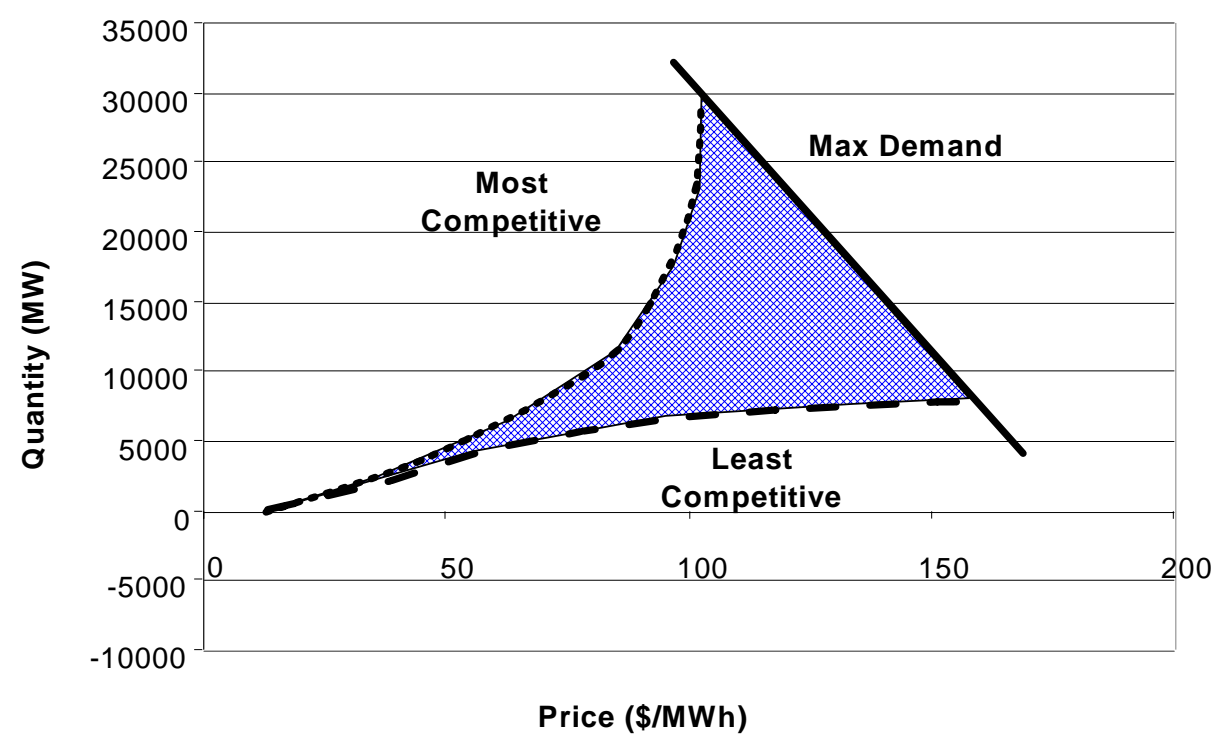

This gives rise to an equilibrium selection problem. ${ }^{4}$ If there are (infinitely) many equilibrium solutions, further restrictions would be needed to identify the predicted equilibrium for the market.

Note the contrast with the competitive solution for price-taking firms. In this case the unique profit maximizing supply function is simply the inverse of the marginal cost function, i.e.,

$$
\begin{aligned}
& S_{i}(p)=\left(C_{i}^{(1)}\right)^{-1}(p)=\frac{p-a_{i}}{C_{i}} \\
& S_{j}(p)=\left(C_{j}^{(1)}\right)^{-1}(p)=\frac{p-a_{j}}{C_{j}} .
\end{aligned}
$$

Since the optimal function is independent of the other firm's offer, the competitive solution is inherently stable.

One approach to narrowing the set of possible strategic equilibria would be to exploit the notion of stability. Given a small perturbation away from an SFE, a locally

Paul D. Klemperer and Margaret A. Meyer. "Supply Function Equilibria in Oligopoly Under Uncertainty,” Econometrica, Vol. 57, No. 6, November 1989, pp. 1243-1277. Richard Green and David M. Newbery, "Competition in the British Electricity Spot Market," Journal of Political Economy, Vol. 100, No. 5, October 1992, pp. 929-953. 
stable equilibrium would imply that the best response to the small perturbation would be closer to the original SFE. An unstable solution would have a best response that might diverge from the original equilibrium.

The appeal of the notion of stability follows from the view that any equilibrium that is not locally stable would be difficult to sustain in a market setting. ${ }^{5}$ Errors in estimation of the supply functions offered by others could lead to best responses that were further from the target equilibrium. For instance, in a dynamic search process where market participants follow a myopic strategy of accepting the last cycle of bids as the best estimate of the current supply function, known as a Cournot adjustment process, it is unlikely that best response iteration could converge to an equilibrium that is not stable. ${ }^{6}$

If few equilibria were stable, then the scope of the equilibrium selection problem would be substantially reduced. Note that the seminal paper on supply function equilibria did not address this question. In particular, Klemperer and Meyer emphasized that "[w]e do not model the process by which markets are brought into equilibrium.", Subsequent work has been largely computational, with various authors reporting simulations that could be interpreted as consistent with the notion that it is difficult to find a stable SFE. ${ }^{8}$ Rudkevich was the first to analyze the problem theoretically and showed that for the particular class of affine supply functions the Cournot adjustment process would converge to the unique, globally stable affine SFE. ${ }^{9}$ Anderson and $\mathrm{Xu}$ analyzed a related model with restrictions to discrete prices and found examples of stable and unstable equilibria. ${ }^{10}$

$5 \quad$ Ziad Younes and Marija Ilic, "Generation Strategies for Gaming Transmission Constraints: Will the Deregulated Electric Power Market be an Oligopoly?”, Decision Support Systems, Vol. 24, 1999, p. 209.

$6 \quad$ Aleksandr Rudekevich, "On the Supply Function Equilibrium and its Application in Electricity Markets,” TCA Working Paper, February 2003, (www.tca-us.com). Note that Cournot adjustment process is applicable to any strategy, not just the similarly named Cournot strategy of offering a fixed quantity.

$7 \quad$ Klemperer and Meyer, (emphasis in original) p. 1245.

$8 \quad$ For example, see Benjamin F. Hobbs, Carolyn B. Metzler, and Jong-Shi Pang, “Strategic Gaming Analysis for Electric Power Systems: An MPEC Approach,” IEEE Transactions on Power Systems, Vol. 15, No. 2, May 2000, pp. 638-645. Christopher J. Day and Derek W. Bunn, "Divestiture of Generation Assets in the Electricity Pool of England and Wales: A Computational Approach to Analyzing Market Power,” Journal of Regulatory Economics, Vol. 19, No. 2, 2001, pp. 123-141. Ross Baldick, Ryan Grant and Edward Kahn, "Theory and Application of Linear Supply Function Equilibrium in Electricity Markets,” Journal of Regulatory Economics, Vol. 25, No. 2, 2004, pp. 143-167.

$9 \quad$ Aleksandr Rudkevich, "Supply Function Equilibrium in Power Markets: Learning All the Way," TCA Technical Paper 1299-1702, 1999, (www.tca-us.com).

10 Edward J. Anderson and Huifu Xu, "Nash Equilibria in Electricity Markets with Discrete Prices,” Australian Graduate School of Management, Working Paper 02-002, June 2002 (forthcoming in Mathematical Methods of Operational Research). 
Baldick and Hogan demonstrated that any unconstrained nonlinear concave or convex SFE would be unstable with respect to a specific tangent perturbation. ${ }^{11}$ In particular, an approximation of a supply function equilibrium that matched the function identically up to a given price and then followed the linear tangent was constructed to show that the best response to this deviation produced a supply function that was further away from the original supply function. This analytical result was consistent with simulations that found unstable unconstrained nonlinear supply functions. By construction, this perturbation would not apply to the affine case where the supply function is by definition coincident with its linear tangent.

The particular tangent perturbation in Baldick and Hogan was suggestive in emphasizing the central role of the slope of the competing supply function in determining the best response. For example, it is immediate from the derivation of the best response that a perturbation of the supply function that merely shifts a supply function intercept would not change the best response. Hence, any SFE would be stable with respect to such parallel shifts.

The tangent perturbation has the defect that it is not twice continuously differentiable at the point of approximation. Hence, the unconstrained best response could not be applied again everywhere as the next response to the perturbation. This would require an analysis that imposed monotonicity constraints on the response, and would no longer satisfy the unconstrained differential equations.

Note that numerical experiments illustrate other problems with a different type of instability, namely numerical instability. For instance, using finite differences to calculate derivatives introduces errors in the determination of the derivatives and soon causes the best response calculation to diverge even from an exact SFE. This is a different problem than considered here. ${ }^{12}$ The present discussion introduces function approximations but assumes the approximations can be implemented with exact arithmetic. Numerical instability is an added and separate difficulty.

The unconstrained solution is the easiest case to address. It would be useful to have a better characterization of perturbations in the space of functions that would define the local properties of any unconstrained SFE and allow for recursive analysis of the limiting behavior of the best response function. The definition of stability is not restricted to a narrow class of perturbations. This raises the question of how better to define the types of perturbations intended and how to analyze the asymptotic behavior of the recursive application of the best response function.

For simplicity, we address here the special case of the symmetric unconstrained SFE where the two firms have the same cost function. This illustrates the basic points.

11 Ross Baldick and William Hogan, "Capacity Constrained Supply Function Equilibrium Models of Electricity Markets: Stability, Non-decreasing Constraints, and Function Space Iterations," Center for Business and Government, August 2002.

12 By contrast with the best response iteration, numerical integration of the known differential equations defining the exact SFEs is a third type of instability. For well behaved SFEs, dealing with this source of error is better understood. 


\section{Symmetric Unconstrained SFE Polynomial Approximation} function as

In the symmetric case, we simplify the problem by restating the best response

$$
\hat{S}(p)=\frac{\gamma+S^{(1)}(p)}{1+c\left(\gamma+S^{(1)}(p)\right)}(p-a) .
$$

We could express the best response as a mapping $(B)$ from an appropriate space of functions $(B: \Sigma \rightarrow \Sigma)$ into itself,

$$
\hat{S}=B(S) .
$$

Then a symmetric unconstrained SFE would be a fixed point of $B$,

$$
S=B(S) \text {. }
$$

A case of special interest is the affine supply function equilibrium with positive slope $(\theta>0)$ defined by the relationship where the function is its own best response:

$$
S(p)=\theta(p-a)=\frac{\gamma+\theta}{1+c(\gamma+\theta)}(p-a) .
$$

Hence,

$$
\begin{aligned}
& \theta=\frac{\gamma+\theta}{1+c(\gamma+\theta)}, \text { or } \\
& \theta=\frac{-\gamma+\sqrt{\gamma^{2}+4 \gamma / c}}{2} .
\end{aligned}
$$

Apparently the affine SFE is a fixed point of the best response mapping. Klemperer and Meyer demonstrate that the affine SFE is the unique unconstrained SFE when the support for the uncertain demand shock is infinite. ${ }^{13}$

Ideally we would like to define $\Sigma$ to simplify the task of specifying a neighborhood $(N)$ of a fixed point $S$ where we could examine the local behavior of the mapping to characterize stability.

It is clear that the best response function has the property of "losing" one degree of differentiability at every application except when the original supply function is infinitely continuously differentiable. Hence, any fixed point that is its own best response must be infinitely continuously differentiable. Thus it appears that $\Sigma$ should be contained in the space $C^{\infty}\left[a, p_{\max }\right]$ of infinitely continuously differentiable functions over a bounded interval of prices. Applying the usual apparatus for analyzing the differential 
of the best response mapping to characterize the local behavior of $B$ would require further restrictions to give $\Sigma \subset C^{\infty}\left[a, p_{\max }\right]$ the structure of a Banach space. ${ }^{14}$

An alternative approach is to characterize an appropriate class of approximations of a supply function and perturbations in terms of this approximation. Consider the general polynomial approximation of degree $n$ for any supply function relative to a price $p_{0}$ :

$$
\tilde{S}(p)=\sum_{j=0}^{n} \frac{\alpha_{j}(S)}{(j) !}\left(p-p_{0}\right)^{j} .
$$

Here the parameters $(\alpha(S))$ would be selected by an appropriate method to give a polynomial approximation of the supply function. The resulting $k$ th derivative of the approximation is

$$
\tilde{S}^{(k)}(p)=\sum_{j=k}^{n} \frac{\alpha_{j}(S)}{(j-k) !}\left(p-p_{0}\right)^{j-k} .
$$

This approximation is continuously differentiable. If the approximation is also monotonic then it is admissible and we could examine equilibrium and stability of the approximation. If the approximation is not monotonic over the range of prices, then the approximation could not be an equilibrium in approximate supply functions. ${ }^{15}$ If the supply function is analytic over a finite open interval, the approximation improves for higher $n .{ }^{16}$ Hence, we focus on the best response for the monotonic approximations.

Given a monotonic polynomial approximation, the best response is

$$
\hat{\tilde{S}}(p)=\frac{\gamma+\tilde{S}^{(1)}(p)}{1+c\left(\gamma+\tilde{S}^{(1)}(p)\right)}(p-a) .
$$

Hence, an approximation SFE would be a fixed point satisfying

14 For example, $C^{\infty}\left[a, p_{\max }\right]$ is not a Banach space under the supremum norm; see Walter Rudin, Functional Analysis, McGraw Hill, second edition, 1991, p.5. For a summary of local stability in Banach spaces see David Ruelle, Elements of Differentiable Dynamics and Bifurcation Theory, Academic Press, 1989, pp. 32-35.

15 Aleksandr Rudkevich raised the point that for some $n$ polynomial approximations of SFEs may not be everywhere monotonic (private communication).

16 For an analysis of supply functions characterized as an infinite power series, see Appendix A of Friedel Bolle, “Competition with Supply and Demand Functions,” Energy Economics, Vol. 23, 2001, pp. 253-277. 


$$
S(p)=\frac{\gamma+\left(\sum_{j=1}^{n} \frac{\alpha_{j}(S)}{(j-1) !}\left(p-p_{0}\right)^{j-1}\right)}{1+c\left(\gamma+\left(\sum_{j=1}^{n} \frac{\alpha_{j}(S)}{(j-1) !}\left(p-p_{0}\right)^{j-1}\right)\right)}(p-a) .
$$

This formulation generalizes the approach of selecting affine approximations to the supply function and calculating the best response to the affine approximation, which is the case of $n=1$. Further, for $n>1$ the polynomial approximation admits perturbations away from the affine SFE and allows tests of stability even in the affine case.

The polynomial approximation is infinitely continuously differentiable with derivatives equal to zero for degree greater than $n$. The best response function also produces an infinitely differentiable function with higher derivatives that are not all equal to zero.

The mapping of the approximation supply function and best response could be interpreted as a representation with imperfect estimation of the other firm's supply function and resulting residual demand. The symmetric case here assumes that both suppliers use the same approximation method as well as have the same cost structure.

Analysis of the approximation supply function reduces to analysis of the mapping of the approximation parameters for the polynomial of degree $n$. Hence, the approximation mapping could be stated as $\left(B: R^{n} \rightarrow R^{n}\right)$ where

$$
\alpha^{k+1}=B\left(\alpha^{k}\right)
$$

A fixed point of the approximation mapping would be a fixed point for the polynomial approximation,

$$
\alpha=B(\alpha) \text {. }
$$

Now the mapping is in the usual finite dimensional space. With an appropriate selection of the approximation rule the mapping will be differentiable. Analysis of stability here reduces to the familiar case allowing application of the Grobman-Hartman theorem for hyperbolic systems. In particular, for any fixed point $\alpha$ of $B$ the behavior of the differential $D B$ evaluated at $\alpha$ characterizes the local stability properties. If the largest modulus of the eigenvalues (i.e., the spectral radius) of the matrix $D B(\alpha)$ is strictly less than one, the system is locally stable and small perturbations converge to the fixed point. If the largest modulus is strictly greater than one, then the system is locally unstable. $^{17}$

$17 \quad$ For a clear summary, see Angel de la Fuente, Mathematical Methods and Models for Economists, Cambridge University Press, 2000, pp. 487-489. When only some of the eigenvalues have modulus greater than one, the differential gives rise to tangents to stable and unstable manifolds. The non-hyperbolic case of the largest eigenvalue modulus equal to one is ambiguous. 
In general, we would anticipate that any rule for selecting the polynomial approximation would have the affine approximation as a special case. Hence, the affine SFE would be a fixed point of the mapping. The neighborhood $N$ of the fixed point would be any polynomial of degree $n$ with derivatives close to the affine case.

\section{Regression Approximation Rule}

One approach to polynomial approximation is through regression on a grid. Suppose that we have a set of prices $\left\{p_{i}\right\}_{i=1, m}$ that defines a grid over the relevant range of prices for the supply function. Then we could choose the coefficients of the polynomial approximation to minimize the sum of the squared differences of the approximation and the supply function at the grid points.

We seek to construct the approximation relative to $p_{0}$ :

$$
\tilde{S}(p)=\sum_{j=0}^{n} \frac{\alpha_{j}}{(j) !}\left(p-p_{0}\right)^{j} .
$$

The coefficients fit a least squares regression rule defined on the grid of $m>>n$ prices and a polynomial of degree $n$ on that grid. The appendix summarizes the particular form of the regression matrix $X$.

Begin with an estimate $\alpha^{k}$ of the derivatives

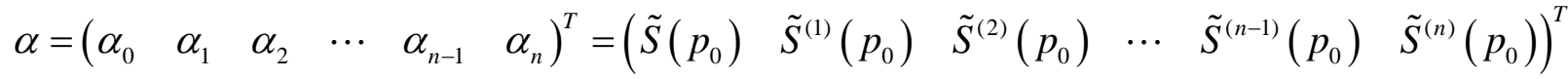

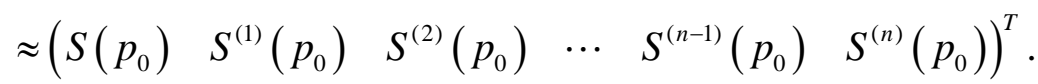

Then the initial polynomial evaluated at the grid point is

$$
\tilde{S}\left(\left\{p_{i}\right\}, \alpha^{k}\right)=X \alpha^{k} \text {. }
$$

Hence, the best response to the approximation is

$$
\hat{\tilde{S}}\left(p_{i}, \alpha^{k}\right)=\frac{\gamma+\tilde{S}^{(1)}\left(p_{i}, \alpha^{k}\right)}{1+c\left(\gamma+\tilde{S}^{(1)}\left(p_{i}, \alpha^{k}\right)\right)}\left(p_{i}-a\right) .
$$

The new least squares estimate of the coefficients would be

$$
\alpha^{k+1}=B\left(\alpha^{k}\right)=\left(X^{T} X\right)^{-1} X^{T}\left(\begin{array}{c}
\hat{\tilde{S}}\left(p_{1}, \alpha^{k}\right) \\
\hat{\tilde{S}}\left(p_{2}, \alpha^{k}\right) \\
\vdots \\
\hat{\tilde{S}}\left(p_{m}, \alpha^{k}\right)
\end{array}\right) \text {. }
$$

Therefore, as shown in the appendix, for any fixed point we have 


$$
\left(X^{T} X\right)^{-1} X^{T}\left(\left(\begin{array}{c}
\hat{\tilde{S}}\left(p_{1}, \alpha\right) \\
\hat{\tilde{S}}\left(p_{2}, \alpha\right) \\
\vdots \\
\tilde{\tilde{S}}\left(p_{m}, \alpha\right)
\end{array}\right)-\left(\begin{array}{c}
\tilde{S}\left(p_{1}, \alpha\right) \\
\tilde{S}\left(p_{2}, \alpha\right) \\
\vdots \\
\tilde{S}\left(p_{m}, \alpha\right)
\end{array}\right)\right)=0
$$

In other words, the difference between the polynomial approximation and its best response must be orthogonal to $X$. This holds trivially at the affine SFE which is its own polynomial approximation and own best response. The condition is the general property of orthogonality of the error that follows from the regression formulation.

Although this orthogonality condition characterizes a necessary condition for any regression approximation equilibrium, it does not appear useful as a means for identifying fixed points other than the obvious affine case. However, the mapping does lend itself easily to calculation of the matrix $D B(\alpha)$ and evaluation of local stability for any candidate solution.

We have from the appendix that the differential matrix is of the form

$$
\begin{aligned}
& D B(\alpha)=\left(X^{T} X\right)^{-1} X^{T} \Delta X \Phi, \\
& \Delta=\operatorname{Diag}\left\{\frac{1}{\left[1+c\left(\gamma+\tilde{S}^{(1)}\left(p_{i}, \alpha\right)\right)\right]^{2}}\right\}_{i=1, m}, \\
& \Phi=\operatorname{Diag}\{i\}_{i=0, n} .
\end{aligned}
$$

The diagonal matrix $\Phi$ contains the index of the degree of the polynomial terms, and this is important in the analysis of the largest eigenvalue modulus.

The difficulty here is that for a nonlinear solution the approximation derivatives are not constant. Hence the diagonal matrix $\Delta$ is on the "inside" and does not admit a simple reduction. Further, the differential matrix is not symmetric and it may have complex eigenvalues. However, given any set of parameters and a fixed point, we can calculate the eigenvalues to test for local stability of the fixed point.

The problem is simpler in the special case of the affine SFE. The unique affine solution $\alpha_{\text {affine }}=\left(\begin{array}{lllll}\theta\left(p_{0}-a\right) & \theta & 0 & \cdots & 0\end{array}\right)^{T}$ is a fixed point for the polynomial regression and has a constant derivative. In this case, the differential matrix becomes 


$$
\begin{aligned}
D B\left(\alpha_{\text {affine }}\right) & =\left(X^{T} X\right)^{-1} X^{T} X \Phi \frac{1}{[1+c(\gamma+\theta)]^{2}} \\
& =\left(\begin{array}{ccccc}
0 & 0 & 0 & \cdots & 0 \\
0 & 1 & 0 & \cdots & 0 \\
0 & 0 & 2 & \cdots & 0 \\
\vdots & \vdots & \vdots & \vdots & \vdots \\
0 & 0 & 0 & \cdots & n
\end{array}\right) \frac{1}{[1+c(\gamma+\theta)]^{2}} .
\end{aligned}
$$

This is a diagonal matrix with the eigenvalues on the diagonal. Apparently we have the largest eigenvalue modulus as

$$
\frac{n}{[1+c(\gamma+\theta)]^{2}} \text {. }
$$

In general, with $\gamma, c>0$ we have $[1+c(\gamma+\theta)]^{2}>1$. Therefore, the affine SFE with polynomial approximation of degree $n=1$ will be locally stable. However, for higher degrees we eventually reach a condition where the affine SFE is not locally stable for the polynomial approximation; i.e. whenever $n$ exceeds the critical threshold

$$
n_{\text {affine }}^{c}=[1+c(\gamma+\theta)]^{2} \text {. }
$$

Numerical experiments confirm this observation. However, before turning to calculations it is useful to look at another approximation that gives more insight about the nature of the fixed points for the approximation supply function equilibrium.

\section{Taylor Series Approximation Rule}

A natural alternative to the regression formulation would be to pick a reference price $\left(p_{0}\right)$ and then form the Taylor series approximation that exactly matches the $n$ derivatives of the supply function at the reference price.

$$
\tilde{S}(p)=\sum_{j=0}^{n} \frac{S^{(j)}\left(p_{0}\right)}{(j) !}\left(p-p_{0}\right)^{j} .
$$

The best response then follows as before with

$$
\hat{\tilde{S}}(p)=\frac{\gamma+\tilde{S}^{(1)}(p)}{1+c\left(\gamma+\tilde{S}^{(1)}(p)\right)}(p-a) .
$$

At a fixed point, this is very close to the regression problem. The results would be the same if the higher derivatives were all zero. Since the higher derivatives are typically not all zero, the two approximations are not quite the same. However, the Taylor approximation offers an advantage in the ability to derive an easily applied analytical expression for the multiple fixed points depending on the derivatives at the 
reference point $\left(p_{0}>a\right){ }^{18}$ This relies on an examination of the derivatives of the best response function.

As shown in the appendix, the $k$ th derivative of the best response is a function of the first $k+1$ derivatives of the approximation function.

For example, for $n=1$

$$
\tilde{\tilde{S}}^{(1)}(p)=\frac{\tilde{S}^{(2)}(p)}{\left[1+c\left(\gamma+\tilde{S}^{(1)}(p)\right)\right]^{2}}(p-a)+\frac{\gamma+\tilde{S}^{(1)}(p)}{1+c\left(\gamma+\tilde{S}^{(1)}(p)\right)} .
$$

For $n=2$

$$
\begin{aligned}
& \hat{\tilde{S}}^{(2)}(p)=\left[\begin{array}{c}
\frac{\tilde{S}^{(3)}(p)}{\left[1+c\left(\gamma+\tilde{S}^{(1)}(p)\right)\right]^{2}} \\
-\frac{2 c\left[\tilde{S}^{(2)}(p) \tilde{S}^{(2)}(p)\right]}{\left[1+c\left(\gamma+\tilde{S}^{(1)}(p)\right)\right]^{3}}
\end{array}\right](p-a) \\
& +\frac{2 \tilde{S}^{(2)}(p)}{\left[1+c\left(\gamma+\tilde{S}^{(1)}(p)\right)\right]^{2}} .
\end{aligned}
$$

and so on.

If we build the polynomial approximation at the reference price $p_{0}>a$, then we can think of this collection of derivatives of the best response as a vector of nonlinear equations creating a mapping of the approximation coefficients for the supply function at the reference point.

Defining equilibrium as a fixed point of this mapping, we would have the form

$$
\alpha=\left(\begin{array}{c}
\alpha_{1} \\
\alpha_{2} \\
\alpha_{3} \\
\alpha_{4} \\
\vdots \\
\alpha_{n+1}
\end{array}\right)=\left(\begin{array}{c}
g(\alpha)) \\
\left.\alpha_{n+1}\right)
\end{array}\right)=\left(\begin{array}{c}
g_{1}\left(\alpha_{1}, \alpha_{2}\right) \\
g_{2}\left(\alpha_{1}, \alpha_{2}, \alpha_{3}\right) \\
g_{3}\left(\alpha_{1}, \alpha_{2}, \alpha_{3}, \alpha_{4}\right) \\
g_{4}\left(\alpha_{1}, \alpha_{2}, \alpha_{3}, \alpha_{4}, \alpha_{5}\right) \\
\vdots \\
g_{n}\left(\alpha_{1}, \alpha_{2}, \alpha_{3}, \alpha_{4}, \alpha_{5}, \cdots, \alpha_{n+1}\right) \\
\alpha_{n+1}
\end{array}\right) .
$$

18 In the regression approximation, the reference price is arbitrary and does not affect the mapping. In the Taylor approximation, the reference price is critical and must be greater than the intercept $a$. At the intercept the exact SFE is not in general analytic. 
Here $\alpha_{i}=S^{(i)}\left(p_{0}\right)$ and the function $g$ is the vector of derivatives of the best response. This system has multiple solutions. Again one solution is the affine case with $\alpha=(\theta, 0,0, \cdots, 0)$.

A related interpretation exploits the simple structure to solve forward for the next derivative if there is an equilibrium, as in

$$
\alpha=\left(\begin{array}{c}
\alpha_{1} \\
\alpha_{2} \\
\alpha_{3} \\
\alpha_{4} \\
\vdots \\
\alpha_{n+1}
\end{array}\right)=\left(\begin{array}{c}
\alpha_{1} \\
h(\alpha)
\end{array}\right)=\left(\begin{array}{c}
\alpha_{1} \\
h_{1}\left(\alpha_{1}\right) \\
h_{2}\left(\alpha_{1}, \alpha_{2}\right) \\
h_{3}\left(\alpha_{1}, \alpha_{2}, \alpha_{3}\right) \\
h_{4}\left(\alpha_{1}, \alpha_{2}, \alpha_{3}, \alpha_{4}\right) \\
\vdots \\
h_{n}\left(\alpha_{1}, \alpha_{2}, \alpha_{3}, \alpha_{4}, \alpha_{5}, \cdots, \alpha_{n}\right)
\end{array}\right) .
$$

Here, $\alpha_{2}=h_{1}\left(\alpha_{1}\right)$ is defined uniquely by $\alpha_{1}=g_{1}\left(\alpha_{1}, h_{1}\left(\alpha_{1}\right)\right)$, and so on. The system is triangular and lends itself to recursive solution. First fix the initial value $\alpha_{1}=S^{(1)}\left(p_{0}\right)$, then solve forward for $S^{(2)}\left(p_{0}\right)=h_{1}\left(S^{(1)}\left(p_{0}\right)\right)$. Repeating this for each stage produces the unique consistent set of derivatives for the SFE with slope $S^{(1)}\left(p_{0}\right)$ at the reference price.

If we applied the recursion to $g$, and started with an exact SFE, we would reproduce the derivatives of the SFE. Note that given $S^{(n+1)}\left(p_{0}\right)$, there may be more than one solution of the system that has this same $(n+1)$ th derivative. For instance, an example below shows that there may be multiple SFEs where some of the derivatives vanish.

Repeated application of the Taylor polynomial approximation of degree $n$ amounts to an iterative application of the system $g$ with the restriction that $S^{(n+1)}\left(p_{0}\right)=0$. If we use the polynomial approximation and iterate the system $g$, then either the iteration converges to an approximation SFE with $S^{(n+1)}\left(p_{0}\right)=0$, or the iteration does not converge.

It follows that the only fixed points for the Taylor polynomial approximation would be those where $S^{(n+1)}\left(p_{0}\right)=0$. These define Taylor approximation SFEs that are unconstrained equilibria for the polynomial approximations of degree $n$. There is always one such approximation equilibrium, the affine SFE. There may be more than one such SFE. We can compute the solutions for all positive values of $S^{(1)}\left(p_{0}\right)$ and examine the zero elements of $h$. With the corresponding degree $n$, these are the candidate limit points for $S^{(1)}\left(p_{0}\right)$ of the approximation iteration. 


\section{SFE Stability \& Approximate Derivatives}

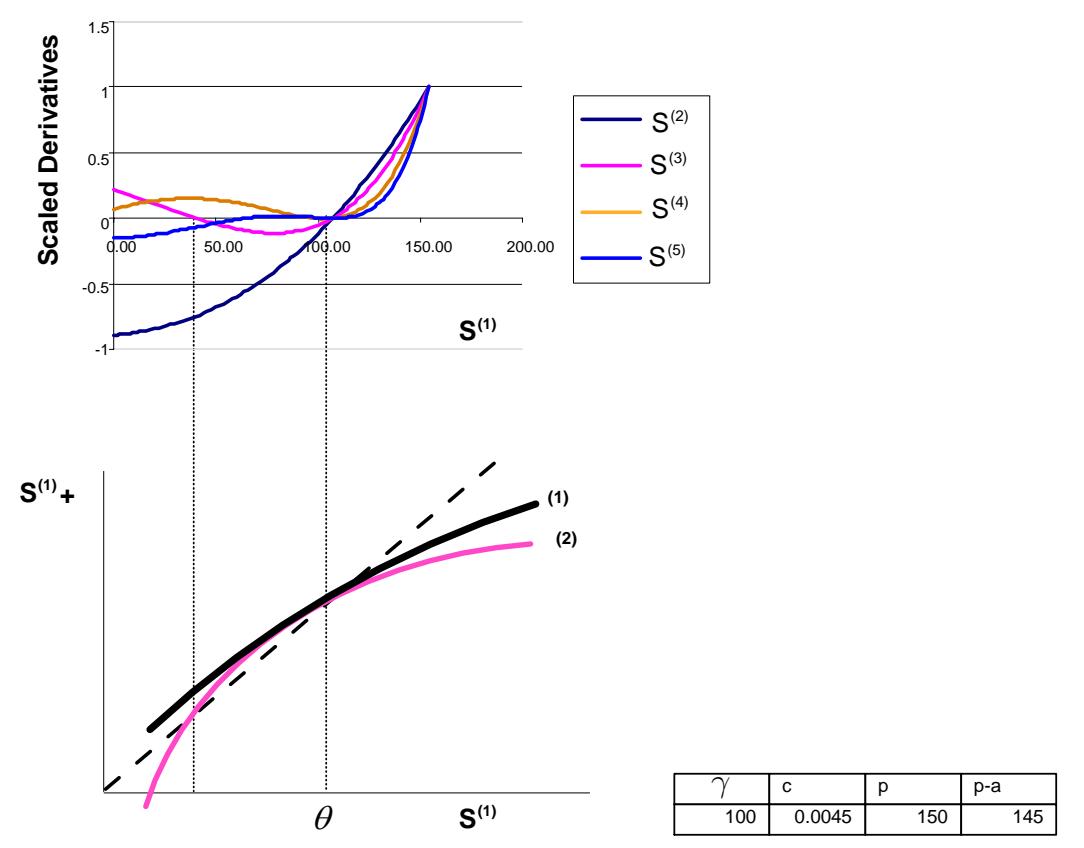

Using an arbitrary set of parameters for demand slope and marginal costs, the top graphic illustrates calculation of approximation SFEs with consistent derivatives using $h$ as $S^{(1)}\left(p_{0}\right)$ varies. All the higher derivatives have value zero at the point $\theta$ which is the unique affine slope. The derivative $S^{(2)}\left(p_{0}\right)$ crosses the zero axis once at this point. The derivative $S^{(3)}\left(p_{0}\right)$ has another zero at $S^{(1)}\left(p_{0}\right)=40.7415$. The derivative $S^{(4)}\left(p_{0}\right)$ has a zero at $S^{(1)}\left(p_{0}\right)=98.166$. And so on.

The bottom graph illustrates a stylized interpretation of the polynomial approximation iteration. The graph labeled (1) shows $g_{1}$ where we fix $S^{(2)}\left(p_{0}\right)=0$. It has one stable solution at $S^{(1)}\left(p_{0}\right)=\theta$. The graph labeled (2) is $g_{1}\left(\alpha_{1}, g_{2}\left(\alpha_{1}, h_{1}\left(\alpha_{1}\right), 0\right)\right)$, where we fix $S^{(3)}\left(p_{0}\right)=0$. It has one fixed point at $S^{(1)}\left(p_{0}\right)=40.7415$ and another at the affine solution at $S^{(1)}\left(p_{0}\right)=\theta$.

We can address the stability of the fixed points for the Taylor approximation by computing the differential of $g$. 


$$
g(\alpha, z)=\left(\begin{array}{c}
g_{1}\left(\alpha_{1}, \alpha_{2}\right) \\
g_{2}\left(\alpha_{1}, \alpha_{2}, \alpha_{3}\right) \\
g_{3}\left(\alpha_{1}, \alpha_{2}, \alpha_{3}, \alpha_{4}\right) \\
g_{4}\left(\alpha_{1}, \alpha_{2}, \alpha_{3}, \alpha_{4}, \alpha_{5}\right) \\
\vdots \\
g_{n}\left(\alpha_{1}, \alpha_{2}, \alpha_{3}, \alpha_{4}, \alpha_{5}, \cdots, z\right)
\end{array}\right)
$$

The polynomial approximation of degree $n$ is the application of $g(\alpha, 0)$. Therefore, the differential is

$$
D g(\alpha, 0)=\left(\begin{array}{cccccc}
\frac{\partial g_{1}}{\partial \alpha_{1}} & \frac{\partial g_{1}}{\partial \alpha_{2}} & 0 & 0 & \ldots & 0 \\
\frac{\partial g_{2}}{\partial \alpha_{1}} & \frac{\partial g_{2}}{\partial \alpha_{2}} & \frac{\partial g_{2}}{\partial \alpha_{3}} & 0 & \ldots & 0 \\
\frac{\partial g_{3}}{\partial \alpha_{1}} & \frac{\partial g_{3}}{\partial \alpha_{2}} & \frac{\partial g_{3}}{\partial \alpha_{3}} & \frac{\partial g_{3}}{\partial \alpha_{4}} & \ldots & 0 \\
\vdots & \vdots & \vdots & \vdots & \vdots & \vdots \\
\frac{\partial g_{n-1}}{\partial \alpha_{1}} & \frac{\partial g_{n-1}}{\partial \alpha_{2}} & \frac{\partial g_{n-1}}{\partial \alpha_{3}} & \ldots & \frac{\partial g_{n-1}}{\partial \alpha_{n-1}} & \frac{\partial g_{n-1}}{\partial \alpha_{n}} \\
\frac{\partial g_{n}}{\partial \alpha_{1}} & \frac{\partial g_{n}}{\partial \alpha_{2}} & \frac{\partial g_{n}}{\partial \alpha_{3}} & \frac{\partial g_{n}}{\partial \alpha_{4}} & \ldots & \frac{\partial g_{n}}{\partial \alpha_{n}}
\end{array}\right) .
$$

We can evaluate this at the affine equilibrium point. As shown in the appendix the largest eigenvalue is again

$$
\frac{n}{[1+c(\gamma+\theta)]^{2}} .
$$

This is the same value for the affine case as found in the regression approximation above. Again for $n$ above the critical threshold,

$$
n_{\text {affine }}^{c}=[1+c(\gamma+\theta)]^{2},
$$

we have the affine solution as locally unstable with respect to the polynomial approximation.

For the general non-affine fixed points we can calculate the differential. For instance, in the case of $n=2$, at a polynomial approximation equilibrium we have 


$$
\begin{aligned}
& \operatorname{Dg}\left(S^{(1)}\left(p_{0}\right), S^{(2)}\left(p_{0}\right), S^{(3)}\left(p_{0}\right)\right) \\
& =\left(\begin{array}{cc}
\frac{2 c S^{(2)}\left(p_{0}\right)\left(p_{0}-a\right)}{\left[1-c\left(\gamma+S^{(1)}\left(p_{0}\right)\right)\right]} & \frac{p_{0}-a}{\left[1+c\left(\gamma+S^{(1)}\left(p_{0}\right)\right)\right]^{2}} \\
\left(\frac{-2 c S^{(2)}\left(p_{0}\right)}{\left.\left[1+c+S^{(1)}\left(p_{0}\right)\right)\right]^{2}}\right)\left(1-\frac{c S^{(2)}\left(p_{0}\right)\left(p_{0}-a\right)}{\left[1+c\left(\gamma+S^{(1)}\left(p_{0}\right)\right)\right]^{3}}\right) & \left.\frac{2-\frac{4 c S^{(2)}\left(p_{0}\right)\left(p_{0}-a\right)}{\left[1+c\left(\gamma+S^{(1)}\left(p_{0}\right)\right)\right]}}{\left[1+c\left(\gamma+S^{(1)}\left(p_{0}\right)\right)\right]^{2}}\right)
\end{array}\right) .
\end{aligned}
$$

Applying the differential to the case of the fixed point for the second order polynomial approximation at $S^{(1)}\left(p_{0}\right)=40.7415$ we find that the largest modulus of the eigenvalues is 1.55 . Hence this fixed point is locally unstable, as confirmed by numerical simulations.

Instability of a nonlinear approximation equilibrium is not always the case. For example, changing the demand slope to $\gamma=10$ yields an affine SFE with $\theta=42.40$ and a quadratic approximation fixed point at $S^{(1)}\left(p_{0}\right)=70.7407$ for which the largest modulus of the mapping differential matrix is 0.70 . This identifies a locally stable nonlinear approximation equilibrium as is confirmed by numerical simulations.

The Taylor approximation is related to the regression approach of the previous section. It is difficult to compute a nonlinear fixed point for the regression approximation. However, a locally stable nonlinear fixed point for the Taylor approximation should be a good starting point in searching numerically for a fixed point of the regression approximation on the grid. Starting with a second derivative consistent with $S^{(1)}\left(p_{0}\right)=70.7407$, regression iterations of the best response find a stable fixed point of the regression approximation polynomial of degree $n=2$ at a slope of $S^{(1)}\left(p_{0}\right)=68.4446$.

Examination of the Taylor approximation for the affine case suggests the nature of the instability for approximations of degree greater than $n_{\text {affine }}^{c}=[1+c(\gamma+\theta)]^{2}$ and points to the related behavior of the more general nonlinear case. As illustrated below, the more elastic the marginal cost curve (i.e., smaller $c$ ) and the more inelastic the demand ( i.e., smaller $\gamma$ ) the lower the critical threshold. Likewise, the more elastic the marginal cost and the more inelastic the demand, the greater the difference between price and marginal cost at any production level; hence, the greater the marginal opportunity cost for the supplier withholding supply. Intuitively, with a larger opportunity cost for withholding, optimization under the best response becomes more sensitive to small changes in the residual demand curve. The optimization exploits the small change of the perturbation. With sufficient non-linearity allowed in the perturbation by including higher degrees in the polynomial, the optimization produces an increase in the nonlinearity of the best response. 
To illustrate this response to a small perturbation, consider the case of a polynomial approximation of degree $n=2$ and the unique affine SFE in $S(p)=\theta(p-a)$. The affine SFE is its own Taylor approximation and a fixed point to the best response. Now suppose we construct an arbitrary perturbation of the polynomial approximation with $|\delta|>0$,

$$
\breve{S}(p)=\theta(p-a)+\frac{\delta^{2}}{2}\left(p-p_{0}\right)^{2} .
$$

With $\delta$ sufficiently small, this quadratic approximation will be arbitrarily close to the affine SFE over the bounded range of prices.

The best response to this perturbation of the approximation function has second derivative

$$
\begin{aligned}
& \hat{\tilde{S}}^{(2)}\left(p_{0}\right)=\left[\begin{array}{c}
\frac{\breve{S}^{(3)}(p)}{\left[1+c\left(\gamma+\breve{S}^{(1)}\left(p_{0}\right)\right)\right]^{2}} \\
\left.-\frac{2 c\left[\breve{S}^{(2)}\left(p_{0}\right) \breve{S}^{(2)}\left(p_{0}\right)\right]}{\left[1+c\left(\gamma+\breve{S}^{(1)}\left(p_{0}\right)\right)\right]^{3}}\right]
\end{array}\right]\left(p_{0}-a\right)+\frac{2 \breve{S}^{(2)}\left(p_{0}\right)}{\left[1+c\left(\gamma+\breve{S}^{(1)}\left(p_{0}\right)\right)\right]^{2}}, \\
& =-\frac{2 c \delta^{2}\left(p_{0}-a\right)}{[1+c(\gamma+\theta)]^{3}}+\frac{2 \delta}{[1+c(\gamma+\theta)]^{2}} \text {. }
\end{aligned}
$$

Hence, we have

$$
\frac{\hat{\tilde{S}}^{(2)}\left(p_{0}\right)}{\delta}=-\frac{2 c \delta\left(p_{0}-a\right)}{[1+c(\gamma+\theta)]^{3}}+\frac{2}{[1+c(\gamma+\theta)]^{2}} .
$$

Therefore, if $\delta$ is sufficiently small and $n_{\text {affine }}^{c}<2$, we have

$$
\frac{\hat{\tilde{S}}^{(2)}\left(p_{0}\right)}{\delta}>1,
$$

and the resulting quadratic approximation to the best response evaluated at the reference price is further from the perturbation from the affine SFE. More generally, for $n>2$ with

$$
\breve{S}(p)=\theta(p-a)+\frac{\delta^{n}}{n !}\left(p-p_{0}\right)^{n},
$$

and with $n_{\text {affine }}^{c}<n$ we obtain

$$
\frac{\hat{\tilde{S}}^{(n)}\left(p_{0}\right)}{\delta}=\frac{n}{[1+c(\gamma+\theta)]^{2}}>1 .
$$


Hence, if $1<n_{\text {affine }}^{c}<n<\infty$ the affine fixed point is locally unstable under the Taylor approximation of degree $n$.

In summary, the Taylor approximation indicates that the continuum of unconstrained exact SFE equilibria reduces to a few possible fixed points with $\tilde{S}^{(n+1)}\left(p_{0}\right)=0$ for the approximation equilibria, one of which is the affine SFE. We see analytically that the affine SFE is locally unstable relative to polynomial approximations of sufficiently large $n$. The form of the general differential matrices also suggests that the same should be true for nonlinear SFEs. However, we do not have an analytical demonstration for other than the case of the affine SFE. Therefore we turn to numerical calculations for various parameters and nonlinear unconstrained SFEs.

\section{Eigenvalue Sensitivity Tests}

The affine SFE provides a simple derivation of the differential for the polynomial approximation mapping. In the case of other possible fixed points, computation of the eigenvalues provides numerical sensitivity tests.

Here we address the case of the regression approach to polynomial approximation. There are three key parameters. First is the affine marginal cost function. The tests here assume an intercept value of $a=\$ 5$ per MWh with different slopes. The slopes are selected according to a benchmark at a marginal cost of $\$ 50$ per MWh, with the base quantities ranging from 5,000 MW to 25,000 MW. Hence, with a quantity benchmark of $10,000 \mathrm{MW}$ we obtain $c=0.0045=(50-5) / 10000$.

The maximum demand at the upper end of the uncertainty range is set at a benchmark price of $\$ 150$ at $60,000 \mathrm{MW}$. If this demand were met by two symmetric firms, each would provide 30,000 MW. The linear slope is set at this demand level to range form 10 to $200 \mathrm{MWh} / \$$. Hence, the demand elasticity at this benchmark level ranges from -0.025 to -0.50 , (e.g., $-0.50=-200 * 150 / 60000$ ).

The simplest case is that of the affine SFE where we have the closed form for the critical threshold for a stable polynomial approximation:

$$
n_{\text {affine }}^{c}=[1+c(\gamma+\theta)]^{2} \text {. }
$$

For values of $n<n_{\text {affine }}^{c}$, a polynomial approximation of degree $n$ at the affine SFE is stable. For values of $n$ greater than the critical value, the affine SFE would be unstable with respect to the polynomial approximations. 


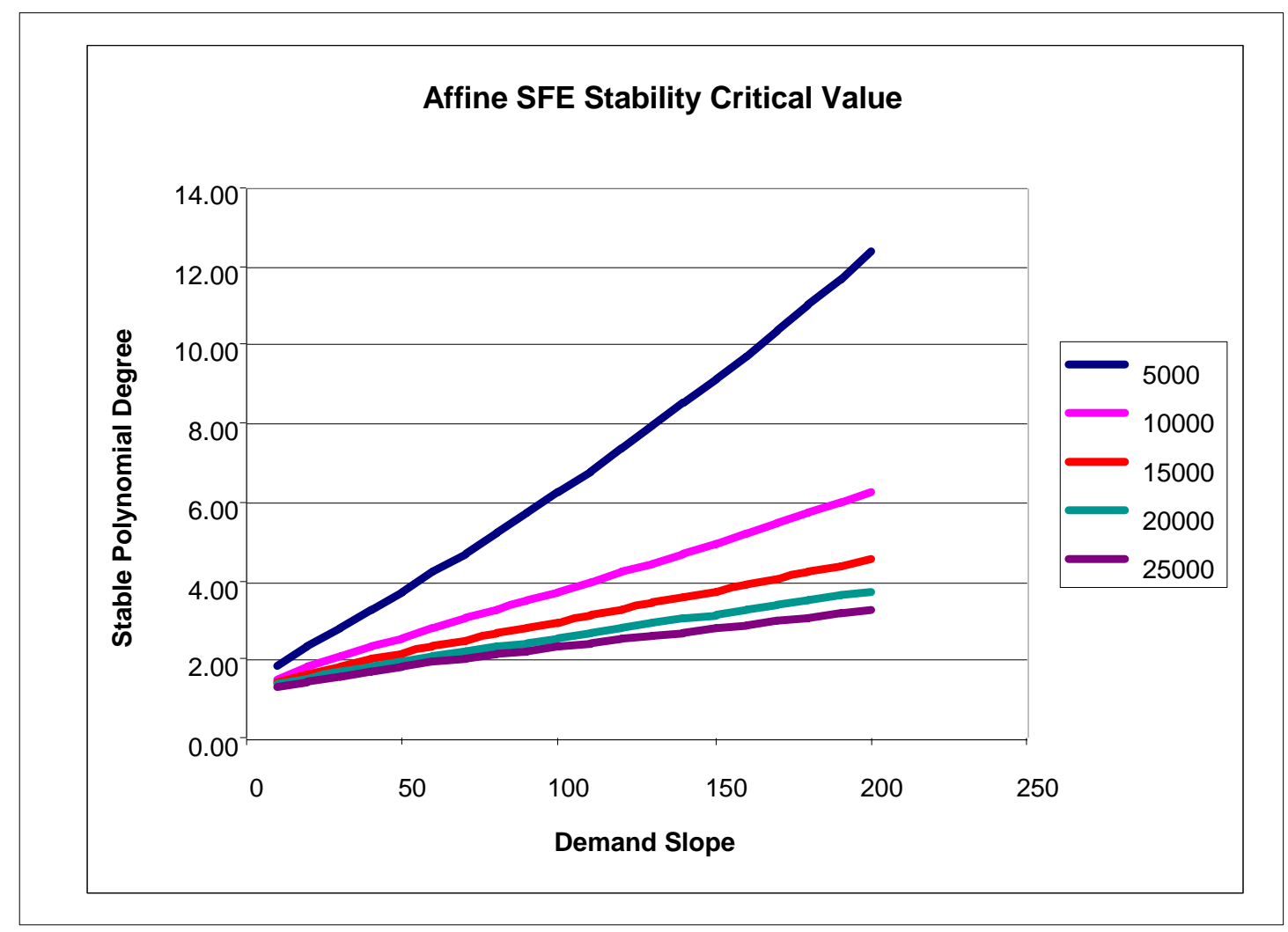

The figure illustrates the affine critical values for the range of sensitivity tests. The horizontal axis varies the demand slope. The separate curves are for different marginal cost benchmark base quantities. The resulting graph is the critical value $n_{\text {affine }}^{c}=[1+c(\gamma+\theta)]^{2}$ below which the polynomial approximation of degree $n<n_{\text {affine }}^{c}$ is stable. Decreasing demand elasticity and decreasing the slope of the marginal cost function tend to make the system unstable sooner.

In the case of nonlinear equilibrium solutions, there is a continuum of possible exact SFEs even though there may be only a few equilibria in the polynomial approximation. However, for every SFE we can calculate the differential of the polynomial mapping and the associated eigenvalues. Two interesting extremes are the least and most competitive SFEs. The least competitive SFE has $S^{(1)}(p)=0$ at the intersection with the maximum demand curve. The most competitive SFE has $S^{(1)}(p)=+\infty$.

Although we do not have a closed form for the exact nonlinear SFEs, as shown in the appendix we can compute the trajectory of an arbitrary SFE. We constructed a grid with $m=500$ points to fit the regression approximations of the respective exact SFEs. Typically the fit of the least competitive SFEs is nearly perfect for $n=3$, with $R^{2} \approx 1$. For example, the first figure below shows the case for a representative case with demand and marginal cost benchmark parameters $(100,10000)$. 

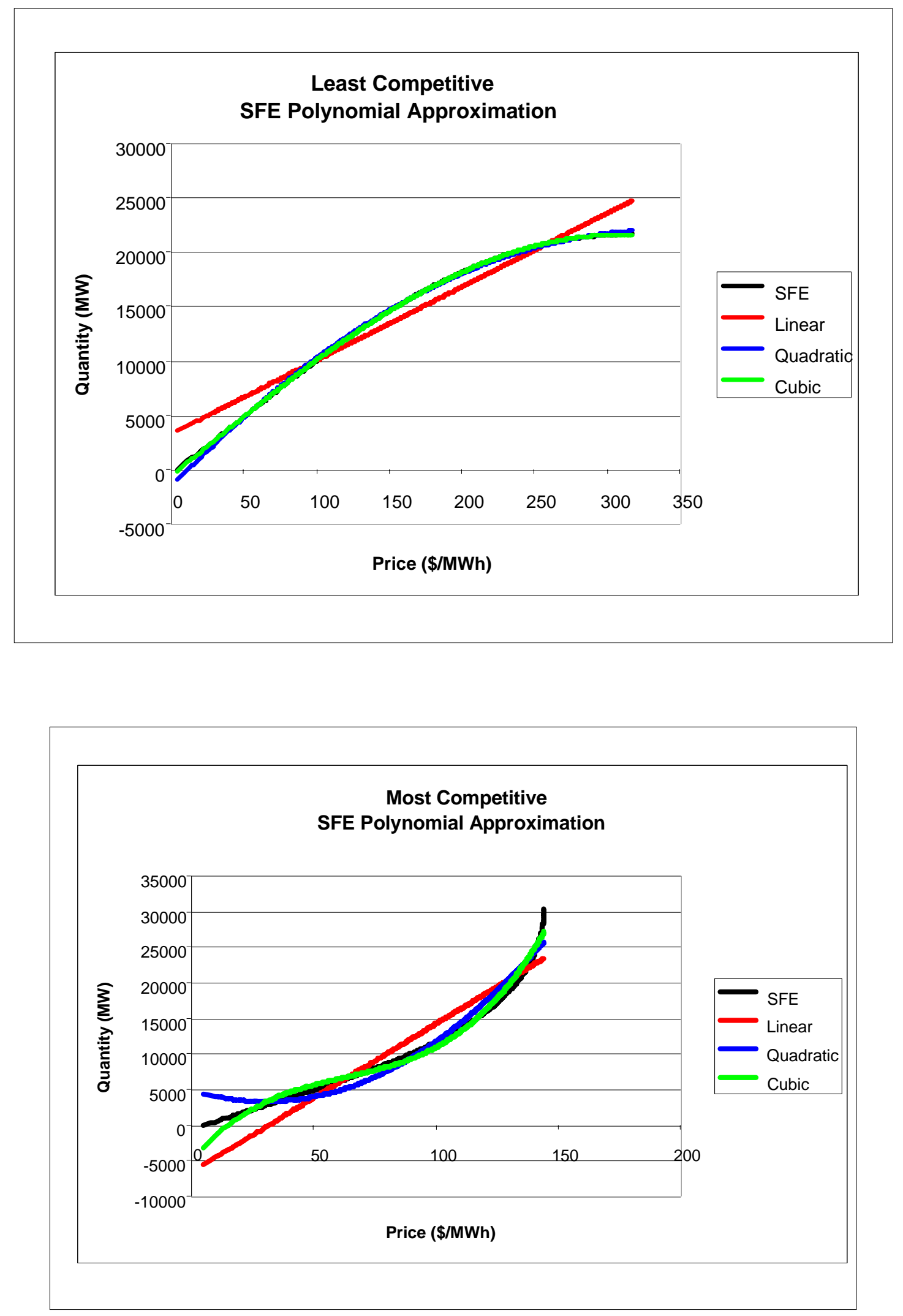
Typically the regression fit for the most competitive case with the same parameters is good but not quite as good as the least competitive case. The second figure above shows the regression for the most competitive case with parameters $(100,10000){ }^{19}$

Given the regression approximation for each degree $n$ we can calculate the eigenvalues of $D B(\alpha)$. A summary of the calculations captures the largest polynomial degree of approximation of that SFE for which an approximation SFE could be stable. (This does not assure that there is a fixed point of the approximation, but only tests for the stability if it is a fixed point.) The calculations for the maximum eigenvalue modulus were done for degrees $n=1,2, \cdots, 10$. Over this range, the largest eigenvalue modulus plot increases approximately linearly in $n$. For $n$ sufficiently large, the regression matrix becomes ill conditioned making it difficult to calculate the eigenvalues accurately. ${ }^{20}$

The analytical result of the critical threshold for stability in the affine case has a numerical analog in the largest integer degree $n$ for which the maximum modulus of the differential eigenvalues is less than one. At or below this degree, a polynomial approximation fixed point, if it exists, would be locally stable. Above this degree, the polynomial approximation would be unstable. A summary of the critical threshold for stability for a range of demand and marginal cost benchmark parameters appears in the following figure for the case of the most competitive SFEs.

19 Note that the quadratic approximation is not everywhere monotonic over the range and could not be an equilibrium.

20 The regression matrix is related to a Vandermonde matrix which is known to be poorly conditioned for large $n$. The eigenvalue calculations were done using Gauss 6.0. 


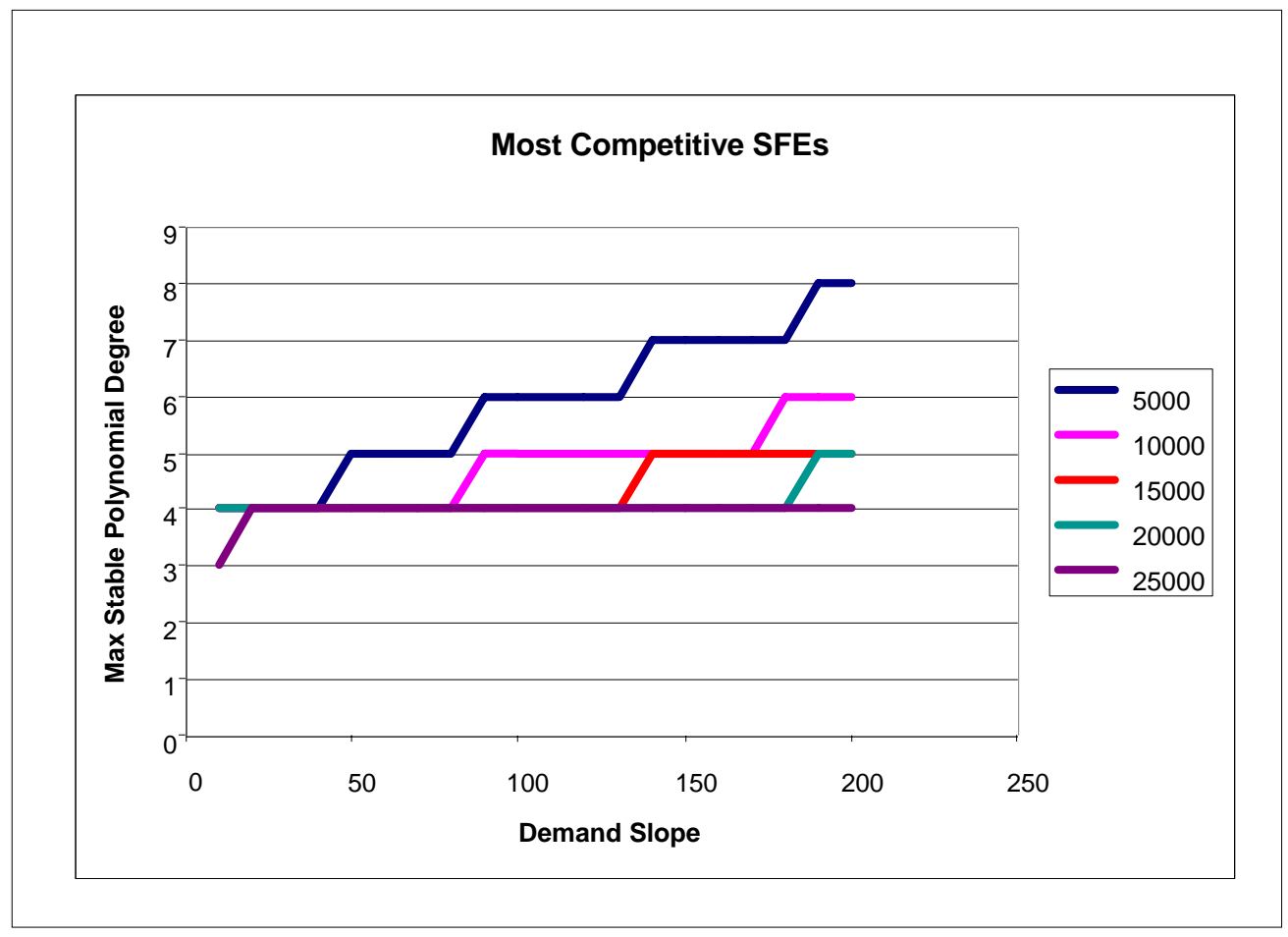

Apparently, for lower values of the demand slopes, the most competitive SFEs are stable for higher degrees of approximation than the affine SFEs. From a market power perspective, however, the most competitive SFEs do not involve much exercise of market power. By construction, at the maximum demand the most competitive SFE has a Lerner index $((p-m c) / p)$ of zero, where price equals marginal cost. ${ }^{21}$ This is similar in outcome to the finding of Anderson and $\mathrm{Xu}$ for models in discrete prices where "if the equilibrium offers are sufficiently close to the generators' marginal costs, then the equilibrium will be stable." 22

The more interesting extreme case is the least competitive SFE. The least competitive nonlinear SFE involves a substantial exercise of market power. At the highest demand level, where the supply function slope is zero, the Lerner index conforms to the pure Cournot strategy result. Since the marginal cost is affine by assumption, the Lerner index is largest at this point.

21 The numerical implementation used a supply function slope of 10,000 as an approximation of infinity. The resulting Lerner indexes at the maximum demand are all less than 0.05.

22 Edward J. Anderson and Huifu Xu, "Nash Equilibria in Electricity Markets with Discrete Prices," Australian Graduate School of Management, Working Paper 02-002, June 2002, p. 1. (forthcoming in Mathematical Methods of Operational Research). 


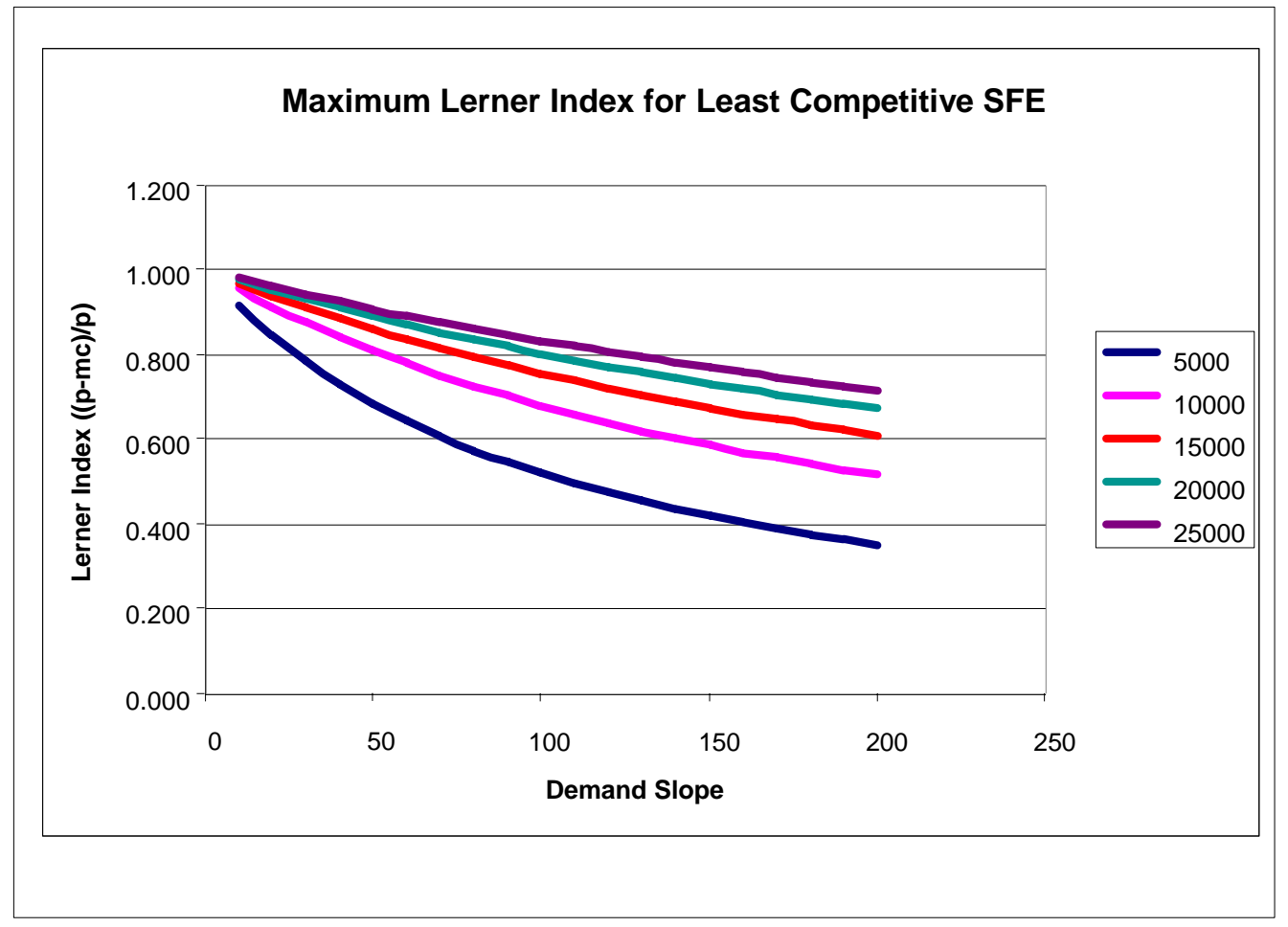

Although this least competitive case would exercise a substantial degree of market power, the following figure shows that the critical threshold of instability is lower than in the case of the affine SFE. 


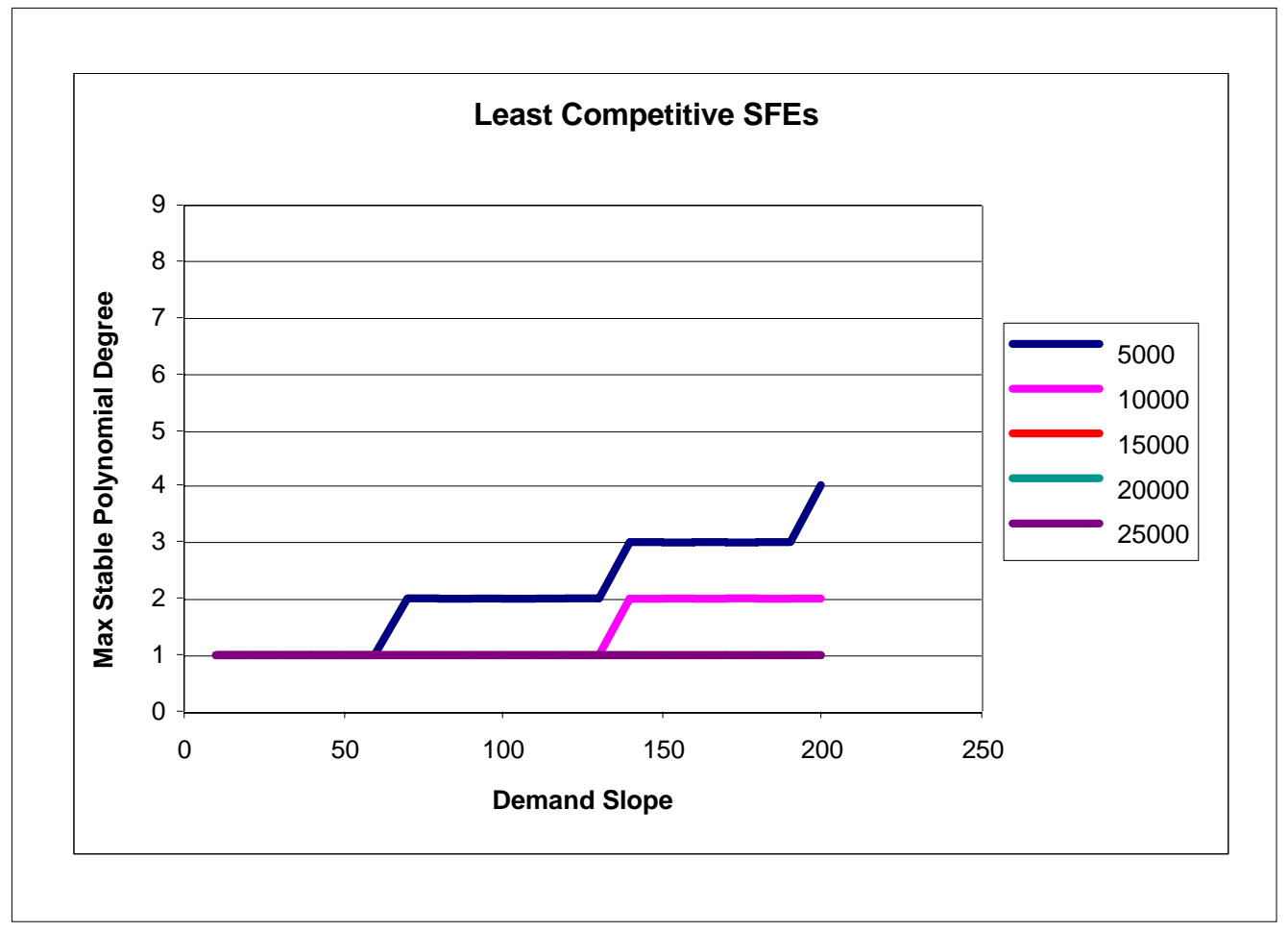

In all but the top two cases with the steepest marginal cost curve, there is no nonlinear polynomial in this range of parameters that provides a stable approximation. And since the linear approximation cannot be a fixed point for any nonlinear SFE, it appears that with a sufficiently flat marginal cost curve the least competitive SFE never gives rise to a stable approximation equilibrium.

\section{Modeling Oligopoly Behavior}

Polynomial approximation has appeal as an implementation of imperfect estimation in a supply function framework where strategic suppliers take the supply function of the other firms as given. Apparently the set of approximation equilibria is small, and is empty for sufficiently large $n$. In addition, use of the symmetric polynomial approximation allows for a fairly general analysis that appears to come arbitrarily close to a full analysis of the exact unconstrained SFE. In this sense, it appears that the unconstrained exact SFE may be chaotic, possessing a continuum of fixed points but no stable fixed points. Of course, approximation at each step is not the same as introducing a single perturbation with exact best response and exact representation for all future iterations. Hence, the results do not prove that the exact SFEs are all unstable. However, if the exact SFE is stable only without any estimation error or with enough restrictions on the form of the supply function to eliminate the polynomials, it is difficult to see how this would be a plausible guide to actual performance of oligopoly markets.

The implication here is that for this form of approximate SFE, virtually any equilibrium would be unstable for high enough degree of the approximation. Furthermore, the tendency towards unstable solutions appears more pronounced for the 
less competitive solutions. If suppliers are not restricting their strategies to known equilibrium positions, it appears as though it would be difficult to achieve any unconstrained equilibrium and especially difficult to achieve an equilibrium that involved the exercise of a substantial degree of market power.

The theoretical and numerical results here are for the simplest of cases, a symmetric equilibrium with affine marginal costs. Relaxing the symmetry assumption to allow for different marginal costs should not have any material effect on the results. This would simply produce a mapping with a block diagonal differential matrix, with one block for each participant that would yield a maximum eigenvalue modulus that grows with the degree $n$.

The more important type of change would be to change the shape of the marginal cost curve. This could then be extended to introduce capacity constraints and impose monotonicity constraints on the supply functions. This would take us beyond the simple unconstrained solution. No longer would the supply function equilibrium be independent of the shape of the distribution of uncertainty. In the case of discrete prices and a uniform distribution of demand, Anderson and $\mathrm{Xu}$ find sufficient conditions that assure local stability when prices are close to marginal costs and then show simulations indicating unstable solutions that do not satisfy these analytical conditions. This leads to their conjecture that "generators in an oligopoly may find it hard to support a noncompetitive equilibrium because of stability issues.",23

The computational work of many authors involving simulations of such systems provides evidence supporting both the computational difficulty of finding equilibria and the constructive computational demonstration that often there is convergence to something. The results here make it less surprising that it is difficult to compute

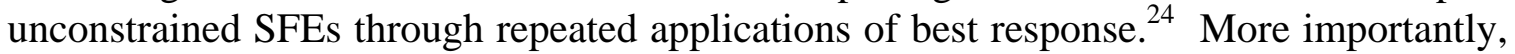
the results reinforce the need for careful attention to the details of the market characterization. Apparently “...seemingly arcane distinctions in assumptions can result in large differences in economic equilibria and disagreements concerning policy implications." 25

In all the models familiar to the authors, once we move from the unconstrained solution as defined by Klemperer and Meyer's results, it is no longer possible to

\footnotetext{
23 Edward J. Anderson and Huifu Xu, "Nash Equilibria in Electricity Markets with Discrete Prices,” Australian Graduate School of Management, Working Paper 02-002, June 2002 (forthcoming in Mathematical Methods of Operational Research), p. 23.

24 Christopher J. Day and Derek W. Bunn, "Divestiture of Generation Assets in the Electricity Pool of England and Wales: A Computational Approach to Analyzing Market Power,” Journal of Regulatory Economics, Vol. 19, No. 2, 2001, p. 128.

25 Carolyn A. Berry, Benjamin F. Hobbs, William A. Meroney, Richard P. O’Neill, William R. Stewart Jr., "Understanding How Market Power Can Arise in Network Competition: A Game Theoretic Approach,” Utilities Policy, Vol. 8, 1999, p. 140. See also, Aleksandr Rudkevich, “On the Supply Function Equilibrium and Its Applications in Electricity Markets,” Tabors Caramanis Associates, February 2003. Julian Barquin, Maroeska G. Boots, Andreas Ehrenmann, Benjamin F. Hobbs, Karsten Neuhoff, and Fieke A.M. Rijkers, "Network-Constrained Models of Liberalized Electricity Markets: the Devil is in the Details,” Cambridge Working Papers in Economics, CWPE 0405, January 2004.
} 
characterize either a best response or a supply function equilibrium simply by examining local results such as the first order conditions at a market equilibrium. In a more general characterization of the supply function equilibrium solution, the model would require a specification of the distribution of uncertainty ${ }^{26}$, multi-settlement contracts ${ }^{27}$, repeated play, the shape of the marginal cost function, ${ }^{28}$ capacity limits, binding monotonicity constraints, bounded rationality, ${ }^{29}$ sub-optimization ${ }^{30}$, and transmission limits ${ }^{31}$, at least.

This result has implications for market monitors who are seen to face a complicated task. If "seemingly arcane distinctions in assumptions can result in large differences in economic equilibria and disagreements concerning policy implications," it would difficult to apply these models as demonstrating the presence or absence of an exercise of market power. Apparently the stability results further reinforce the notion that the properties of these oligopoly models are not well understood and equilibrium solutions in the model may not correspond to market outcomes. Even seemingly wellbehaved existence results for equilibria when we can impose the form of the equilibrium solution are not accompanied by stability properties with imperfect estimation of the functional form and iteration of the best response. The conclusion supports the need for continuing research on oligopoly models that incorporate added details and can be validated against market data.

26 Edward J. Anderson and Huifu Xu, "Necessary and Sufficient Conditions for Optimal Offers in Electricity Markets,” SIAM Journal of Control and Optimization, Vol. 41, No. 4, 2002, pp. 1212-1228.

27 Steven C. Anderson, “Analyzing Strategic Interaction In Multi-Settlement Electricity Markets: A Closed-Loop Supply Function Equilibrium Model”, Harvard University Ph. D. Dissertation, June 2004.

$28 \quad$ Ross Baldick, Ryan Grant and Edward Kahn, “Theory and Application of Linear Supply Function Equilibrium in Electricity Markets,” Journal of Regulatory Economics, Vol. 25, No. 2, 2004, pp. 143-167.

29 Christopher J. Day and Derek W. Bunn, "Divestiture of Generation Assets in the Electricity Pool of England and Wales: A Computational Approach to Analyzing Market Power,” Journal of Regulatory Economics, Vol. 19, No. 2, 2001, p. 129.

30 Edward J. Anderson and Huifu $\mathrm{Xu}$, “ $\varepsilon$-Optimal Bidding in an Electricity Market with Discontinuous Market Distribution Functions,” Australian Graduate School of Management, April 2003.

31 C. J. Day, B. F. Hobbs, and J. S. Pang, “Oligopolistic Competition in Power Networks: A Conjectured Supply Function Approach,” IEEE Transactions on Power Systems, Vol. 17, No. 3, 2002, pp. 597-606. 


\section{Appendix}

The regression and Taylor series polynomial approximations give rise to slightly different characterizations of approximation fixed points and the differential at a fixed point. The two approaches complement each other. The Taylor approximation identifies candidate fixed points, but is less intuitive as an approximation technique since it requires exact derivatives and depends on the choice of reference price. The regression approximation is more plausible as an approximation technique but it is more difficult to identify its nonlinear fixed points. The two models have exactly the same theoretical results for local stability conditions for the affine SFE and similar computational results for nonlinear SFEs.

\section{Regression Model}

We seek to construct the approximation relative to $p_{0}$

$$
\tilde{S}(p)=\sum_{j=0}^{n} \frac{\alpha_{j}}{(j) !}\left(p-p_{0}\right)^{j} .
$$

Select the coefficients to fit a least squares regression rule defined on a grid of $m>>n$ prices and a polynomial of degree $n$ on that grid. Let the matrix of the polynomial terms across the grid be:

$$
X=\left(\begin{array}{cccccc}
1 & p_{1}-p_{0} & \frac{\left(p_{1}-p_{0}\right)^{2}}{2} & \cdots & \frac{\left(p_{1}-p_{0}\right)^{n-1}}{(n-1) !} & \frac{\left(p_{1}-p_{0}\right)^{n}}{(n) !} \\
1 & p_{2}-p_{0} & \frac{\left(p_{2}-p_{0}\right)^{2}}{2} & \cdots & \frac{\left(p_{2}-p_{0}\right)^{n-1}}{(n-1) !} & \frac{\left(p_{2}-p_{0}\right)^{n}}{(n) !} \\
1 & p_{3}-p_{0} & \frac{\left(p_{3}-p_{0}\right)^{2}}{2} & \cdots & \frac{\left(p_{3}-p_{0}\right)^{n-1}}{(n-1) !} & \frac{\left(p_{3}-p_{0}\right)^{n}}{(n) !} \\
\vdots & \vdots & \vdots & \vdots & \vdots & \vdots \\
1 & p_{m-1}-p_{0} & \frac{\left(p_{m-1}-p_{0}\right)^{2}}{2} & \cdots & \frac{\left(p_{m-1}-p_{0}\right)^{n-1}}{(n-1) !} & \frac{\left(p_{m-1}-p_{0}\right)^{n}}{(n) !} \\
1 & p_{m}-p_{0} & \frac{\left(p_{m}-p_{0}\right)^{2}}{2} & \cdots & \frac{\left(p_{m}-p_{0}\right)^{n-1}}{(n-1) !} & \frac{\left(p_{m}-p_{0}\right)^{n}}{(n) !}
\end{array}\right) .
$$

And let the matrix of derivatives of the polynomial terms be 


$$
Z=\left(\begin{array}{cccccc}
0 & 1 & p_{1}-p_{0} & \cdots & \frac{\left(p_{1}-p_{0}\right)^{n-2}}{(n-2) !} & \frac{\left(p_{1}-p_{0}\right)^{n-1}}{(n-1) !} \\
0 & 1 & p_{2}-p_{0} & \cdots & \frac{\left(p_{2}-p_{0}\right)^{n-2}}{(n-2) !} & \frac{\left(p_{2}-p_{0}\right)^{n-1}}{(n-1) !} \\
0 & 1 & p_{3}-p_{0} & \cdots & \frac{\left(p_{3}-p_{0}\right)^{n-2}}{(n-2) !} & \frac{\left(p_{3}-p_{0}\right)^{n-1}}{(n-1) !} \\
\vdots & \vdots & \vdots & \vdots & \vdots & \vdots \\
0 & 1 & p_{m-1}-p_{0} & \cdots & \frac{\left(p_{m-1}-p_{0}\right)^{n-2}}{(n-2) !} & \frac{\left(p_{m-1}-p_{0}\right)^{n-1}}{(n-1) !} \\
0 & 1 & p_{m}-p_{0} & \cdots & \frac{\left(p_{m}-p_{0}\right)^{n-2}}{(n-1) !} & \frac{\left(p_{m}-p_{0}\right)^{n-1}}{(n-1) !}
\end{array}\right) .
$$

Begin with an estimate $\alpha^{k}$ of the derivatives

$$
\begin{aligned}
& \alpha=\left(\begin{array}{llllll}
\alpha_{0} & \alpha_{1} & \alpha_{2} & \cdots & \alpha_{n-1} & \alpha_{n}
\end{array}\right)^{T}=\left(\begin{array}{llllll}
\tilde{S}\left(p_{0}\right) & \tilde{S}^{(1)}\left(p_{0}\right) & \tilde{S}^{(2)}\left(p_{0}\right) & \cdots & \tilde{S}^{(n-1)}\left(p_{0}\right) & \tilde{S}^{(n)}\left(p_{0}\right)
\end{array}\right)^{T} \\
& \approx\left(\begin{array}{llllll}
S\left(p_{0}\right) & S^{(1)}\left(p_{0}\right) & S^{(2)}\left(p_{0}\right) & \cdots & S^{(n-1)}\left(p_{0}\right) & \left.S^{(n)}\left(p_{0}\right)\right)^{T} .
\end{array}\right.
\end{aligned}
$$

Then

$$
\tilde{S}\left(\left\{p_{i}\right\}\right)=X \alpha .
$$

For each $i=1,2, \cdots, m$ and with $Z_{i}$. as the $i$-th row of $Z$, we have the slope of the polynomial approximation at iteration $k$ as:

$$
\tilde{S}^{(1)}\left(p_{i}\right)=Z_{i \cdot} \alpha^{k} .
$$

Hence, the best response to the approximation is

$$
\hat{\tilde{S}}\left(p_{i}\right)=\frac{\gamma+Z_{i \cdot} \alpha^{k}}{1+c\left(\gamma+Z_{i} \cdot \alpha^{k}\right)}\left(p_{i}-a\right) .
$$

The new least squares estimate of the coefficients is 


$$
\alpha^{k+1}=B\left(\alpha^{k}\right)=\left(X^{T} X\right)^{-1} X^{T}\left(\begin{array}{c}
\hat{\tilde{S}}\left(p_{1}\right) \\
\tilde{\tilde{S}}\left(p_{2}\right) \\
\vdots \\
\tilde{\tilde{S}}\left(p_{m}\right)
\end{array}\right)=\left(X^{T} X\right)^{-1} X^{T}\left(\begin{array}{c}
\frac{\gamma+Z_{1} \cdot \alpha^{k}}{1+c\left(\gamma+Z_{1} \cdot \alpha^{k}\right)}\left(p_{1}-a\right) \\
\frac{\gamma+Z_{2} \cdot \alpha^{k}}{1+c\left(\gamma+Z_{2} \cdot \alpha^{k}\right)}\left(p_{2}-a\right) \\
\vdots \\
\frac{\gamma+Z_{m} \cdot \alpha^{k}}{1+c\left(\gamma+Z_{m} \cdot \alpha^{k}\right)}\left(p_{m}-a\right)
\end{array}\right) \text {. }
$$

Therefore, any fixed point of this polynomial approximation must have

$$
\alpha=B(\alpha)=\left(X^{T} X\right)^{-1} X^{T}\left(\begin{array}{c}
\frac{\gamma+Z_{1} \cdot \alpha}{1+c\left(\gamma+Z_{1} \cdot \alpha\right)}\left(p_{1}-a\right) \\
\frac{\gamma+Z_{2 \cdot} \alpha}{1+c\left(\gamma+Z_{2} \cdot \alpha\right)}\left(p_{2}-a\right) \\
\vdots \\
\frac{\gamma+Z_{m} \cdot \alpha}{1+c\left(\gamma+Z_{m} \cdot \alpha\right)}\left(p_{m}-a\right)
\end{array}\right) .
$$

Hence, we can rewrite this as

$$
\left(X^{T} X\right)^{-1} X^{T}\left(\begin{array}{c}
\frac{\gamma+Z_{1} \cdot \alpha}{1+c\left(\gamma+Z_{1} \cdot \alpha\right)}\left(p_{1}-a\right) \\
\frac{\gamma+Z_{2 \cdot} \alpha}{1+c\left(\gamma+Z_{2} \cdot \alpha\right)}\left(p_{2}-a\right) \\
\vdots \\
\frac{\gamma+Z_{m} \cdot \alpha}{1+c\left(\gamma+Z_{m} \cdot \alpha\right)}\left(p_{m}-a\right)
\end{array}\right)-\alpha=0
$$

Therefore, 


$$
\begin{gathered}
\left(X^{T} X\right)^{-1} X^{T}\left(\left(\begin{array}{c}
\left.\frac{\gamma+Z_{1} \cdot \alpha}{1+c\left(\gamma+Z_{1} \cdot \alpha\right)}\left(p_{1}-a\right)\right) \\
\frac{\gamma+Z_{2 \cdot} \alpha}{1+c\left(\gamma+Z_{2} \cdot \alpha\right)}\left(p_{2}-a\right) \\
\vdots \\
\frac{\gamma+Z_{m} \cdot \alpha}{1+c\left(\gamma+Z_{m} \alpha\right)}\left(p_{m}-a\right)
\end{array}\right)-X \alpha\right) \\
=\left(X^{T} X\right)^{-1} X^{T}\left(\left(\begin{array}{c}
\hat{\tilde{S}}\left(p_{1}\right) \\
\tilde{\tilde{S}}\left(p_{2}\right) \\
\vdots \\
\hat{\tilde{S}}\left(p_{m}\right)
\end{array}\right)-\left(\begin{array}{c}
\tilde{S}\left(p_{1}\right) \\
\tilde{S}\left(p_{2}\right) \\
\vdots \\
\tilde{S}\left(p_{m}\right)
\end{array}\right)\right)=0 .
\end{gathered}
$$

In other words, the difference between the polynomial approximation and its best response must be orthogonal to $X$. This holds trivially at the affine SFE which is its own polynomial approximation and own best response. It is a general property that follows from the regression formulation. It is not obvious how to use this characterization to identify a nonlinear fixed point.

However, we can test the stability of any fixed point by calculating the spectral radius or largest eigenvalue of the differential

$$
D B(\alpha)=\left(X^{T} X\right)^{-1} X^{T}\left(\begin{array}{ccccc}
\frac{Z_{11}\left(p_{1}-a\right)}{\left[1+c\left(\gamma+Z_{1} \cdot \alpha\right)\right]^{2}} & \frac{Z_{12}\left(p_{1}-a\right)}{\left[1+c\left(\gamma+Z_{1} \cdot \alpha\right)\right]^{2}} & \cdots & \frac{Z_{1(n+1)}\left(p_{1}-a\right)}{\left[1+c\left(\gamma+Z_{1} \cdot \alpha\right)\right]^{2}} \\
\frac{Z_{21}\left(p_{2}-a\right)}{\left[1+c\left(\gamma+Z_{2} \cdot \alpha\right)\right]^{2}} & \frac{Z_{22}\left(p_{2}-a\right)}{\left[1+c\left(\gamma+Z_{2} \cdot \alpha\right)\right]^{2}} & \cdots & \frac{Z_{2(n+1)}\left(p_{2}-a\right)}{\left[1+c\left(\gamma+Z_{2} \cdot \alpha\right)\right]^{2}} \\
\frac{Z_{m 1}\left(p_{m}-a\right)}{\left[1+c\left(\gamma+Z_{m} \cdot \alpha\right)\right]^{2}} & \frac{Z_{m 2}\left(p_{m}-a\right)}{\left[1+c\left(\gamma+Z_{m} \cdot \alpha\right)\right]^{2}} & \cdots & \frac{Z_{m(n+1)}\left(p_{m}-a\right)}{\left[1+c\left(\gamma+Z_{m} \cdot \alpha\right)\right]^{2}}
\end{array}\right) .
$$

The choice of $p_{0}$ is arbitrary and does not affect the least squares fit of the polynomial. With $p_{0}=a$ the differential is equivalent to 


$$
D B(\alpha)=\left(X^{T} X\right)^{-1} X^{T}\left(\begin{array}{ccccc}
\frac{1}{\left[1+c\left(\gamma+Z_{1} \alpha\right)\right]^{2}} & 0 & 0 & 0 \\
0 & \frac{1}{\left[1+c\left(\gamma+Z_{2} \alpha\right)\right]^{2}} & 0 & 0 \\
\vdots & \vdots & \vdots \\
0 & 0 & \cdots & \frac{1}{\left[1+c\left(\gamma+Z_{m} \alpha\right)\right]^{2}}
\end{array}\right)\left(\begin{array}{cccc}
0 & p_{1}-a & \cdots & \frac{\left(p_{1}-a\right)^{n}}{(n-1) !} \\
0 & p_{2}-a & \cdots & \frac{\left(p_{2}-a\right)^{n}}{(n-1) !} \\
& & & \vdots \\
0 & p_{m}-a & \cdots & \frac{\left(p_{m}-a\right)^{n}}{(n-1) !}
\end{array}\right) .
$$

In other words, we have

$$
\begin{aligned}
& D B(\alpha)=\left(X^{T} X\right)^{-1} X^{T} \Delta X \Phi, \\
& \Delta=\operatorname{Diag}\left\{\frac{1}{\left[1+c\left(\gamma+\tilde{S}^{(1)}\left(p_{i}, \alpha\right)\right)^{2}\right.}\right\}_{i=1, m}, \\
& \Phi=\operatorname{Diag}\{i\}_{i=0, n} .
\end{aligned}
$$

In general, this differential matrix is not symmetric and it may have complex eigenvalues. The matrix is singular, but the singularity arises only because of the constant term in the regression which is not of interest for the stability analysis. Formally we apply the Grobman-Hartman theorem to the nonsingular submatrix for the parameters other than the intercept in the polynomial approximation. ${ }^{32}$ Given a set of parameters and a fixed point, we can calculate the eigenvalues to test for the local stability of the fixed point.

The problem is simpler in the special case of the affine SFE. The unique affine solution $\alpha_{\text {affine }}=\left(\begin{array}{lllll}\theta\left(p_{0}-a\right) & \theta & 0 & \cdots & 0\end{array}\right)^{T}$ is a fixed point for the polynomial regression. In this case, the diagonal matrix is constant and the common value factors out yielding the differential as

$$
\begin{aligned}
D B\left(\alpha_{\text {affine }}\right) & =\left(X^{T} X\right)^{-1} X^{T} X \Phi \frac{1}{[1+c(\gamma+\theta)]^{2}} \\
& =\left(\begin{array}{ccccc}
0 & 0 & 0 & \cdots & 0 \\
0 & 1 & 0 & \cdots & 0 \\
0 & 0 & 2 & \cdots & 0 \\
\vdots & \vdots & \vdots & \vdots & \vdots \\
0 & 0 & 0 & \cdots & n
\end{array}\right) \frac{1}{[1+c(\gamma+\theta)]^{2}} .
\end{aligned}
$$

32 David Ruelle, Elements of Differentiable Dynamics and Bifurcation Theory, Academic Press, 1989, p. 21. 
This is a diagonal matrix with the real eigenvalues on the diagonal. Apparently we have the largest eigenvalue modulus as

$$
\frac{n}{[1+c(\gamma+\theta)]^{2}} \text {. }
$$

In general, with $\gamma, c>0$ we have $[1+c(\gamma+\theta)]^{2}>1$. Hence, the affine approximation with a polynomial of degree $n=1$ will be locally stable at the affine SFE, consistent with the results of Rudkevich and of Baldick, Grant, and Kahn. ${ }^{33}$ However, for polynomial degrees greater than $n_{\text {affine }}^{c}=[1+c(\gamma+\theta)]^{2}$ we reach a condition where the affine SFE is not locally stable for the polynomial approximation.

\section{Taylor Approximation Model}

The Taylor series approximation at $p_{0}>a$ is

$$
\tilde{S}(p)=\sum_{j=0}^{n} \frac{S^{(j)}\left(p_{0}\right)}{(j) !}\left(p-p_{0}\right)^{j} .
$$

The corresponding unconstrained best response is

$$
\hat{\tilde{S}}(p)=\frac{\gamma+\tilde{S}^{(1)}(p)}{1+c\left(\gamma+\tilde{S}^{(1)}(p)\right)}(p-a) .
$$

Taking the successive derivatives, we have:

Aleksandr Rudkevich, "Supply Function Equilibrium in Power Markets: Learning All the Way," TCA Technical Paper 1299-1702, 1999, (www.tca-us.com). Ross Baldick, Ryan Grant, and Edward P. Kahn. "Linear supply function equilibrium: Generalizations, application, and limitations." University of California Energy Institute POWER Paper PWP-078, (www.ucei.berkeley.edu/ucei/PDF/pwp078.pdf), August 2000. 
For $n=1$

$$
\hat{\tilde{S}}^{(1)}(p)=\frac{\tilde{S}^{(2)}(p)}{\left[1+c\left(\gamma+\tilde{S}^{(1)}(p)\right)\right]^{2}}(p-a)+\frac{\gamma+\tilde{S}^{(1)}(p)}{1+c\left(\gamma+\tilde{S}^{(1)}(p)\right)} .
$$

For $n=2$

$$
\begin{aligned}
& \hat{\tilde{S}}^{(2)}(p)=\left[\begin{array}{c}
\frac{\tilde{S}^{(3)}(p)}{\left[1+c\left(\gamma+\tilde{S}^{(1)}(p)\right)\right]^{2}} \\
-\frac{2 c\left[\tilde{S}^{(2)}(p) \tilde{S}^{(2)}(p)\right]}{\left[1+c\left(\gamma+\tilde{S}^{(1)}(p)\right)\right]^{3}}
\end{array}\right](p-a) \\
& +\frac{2 \tilde{S}^{(2)}(p)}{\left[1+c\left(\gamma+\tilde{S}^{(1)}(p)\right)\right]^{2}} .
\end{aligned}
$$

For $n=3$

$$
\begin{aligned}
& \hat{\tilde{S}}^{(3)}(p)=\left[\begin{array}{l}
\frac{\tilde{S}^{(4)}(p)}{\left[1+c\left(\gamma+\tilde{S}^{(1)}(p)\right)\right]^{2}} \\
-\frac{(2+2 \cdot 2) c \tilde{S}^{(3)}(p) \tilde{S}^{(2)}(p)}{\left[1+c\left(\gamma+\tilde{S}^{(1)}(p)\right)\right]^{3}} \\
+\frac{2 \cdot 3 c^{2}\left[\tilde{S}^{(2)}(p) \tilde{S}^{(2)}(p) \tilde{S}^{(2)}(p)\right]}{\left[1+c\left(\gamma+\tilde{S}^{(1)}(p)\right)\right]^{4}}
\end{array}\right](p-a) \\
& +\frac{3 \tilde{S}^{(3)}(p)}{\left[1+c\left(\gamma+\tilde{S}^{(1)}(p)\right)\right]^{2}} \\
& -\frac{(2+2 \cdot 2) c\left[\tilde{S}^{(2)}(p) \tilde{S}^{(2)}(p)\right]}{\left[1+c\left(\gamma+\tilde{S}^{(1)}(p)\right)\right]^{3}} .
\end{aligned}
$$


For $n=4$

$$
\left.\begin{array}{l}
\tilde{\tilde{S}}^{(4)}(p)=\left[\begin{array}{l}
\frac{\tilde{S}^{(5)}(p)}{\left[1+c\left(\gamma+\tilde{S}^{(1)}(p)\right)\right]^{2}} \\
-\frac{(2+(2+2 \cdot 2)) c \tilde{S}^{(4)}(p) \tilde{S}^{(2)}(p)+(2+2 \cdot 2) c\left[\tilde{S}^{(3)}(p) \tilde{S}^{(3)}(p)\right]}{\left[1+c\left(\gamma+\tilde{S}^{(1)}(p)\right)\right]^{3}} \\
+\frac{((4 \cdot(2+2 \cdot 2)+3(2 \cdot 3))) c^{2} \tilde{S}^{(3)}(p)\left[\tilde{S}^{(2)}(p) \tilde{S}^{(2)}(p)\right]}{\left[1+c\left(\gamma+\tilde{S}^{(1)}(p)\right)\right]^{4}}
\end{array}\right. \\
+\frac{2 \cdot 3 \cdot 4 c^{3}\left[\tilde{S}^{(2)}(p) \tilde{S}^{(2)}(p) \tilde{S}^{(2)}(p) \tilde{S}^{(2)}(p)\right]}{-\left[1+c\left(\gamma+\tilde{S}^{(1)}(p)\right)\right]^{5}} \\
{\left[1+c\left(\gamma+\tilde{S}^{(1)}(p)\right)\right]^{2}} \\
-\frac{(2+2 \cdot 2+3 \cdot 3+2 \cdot(2+2 \cdot 2)) c \tilde{S}^{(3)}(p) \tilde{S}^{(2)}(p)}{\left[1+c\left(\gamma+\tilde{S}^{(1)}(p)\right)\right]^{3}}
\end{array}\right](p-a)
$$

We can derive the general form of the derivatives. For $n=2,3, \cdots$, we can show that

$$
\hat{\tilde{S}}^{(n)}(p)=\left[\sum_{k=1}^{n} \frac{\sum_{\left\{j_{i}\right\} \in J(n, k)} a_{n, k,\left\{j_{i}\right\}} \prod_{i=1}^{k} \tilde{S}^{\left(j_{i}\right)}(p)}{\left[1+c\left(\gamma+\tilde{S}^{\prime}(p)\right)\right]^{k+1}}\right](p-a)+\sum_{k=1}^{n-1} \frac{\sum_{\left\{j_{i}\right\} \in J(n-1, k)} b_{n, k,\left\{j_{i}\right\}} \prod_{i=1}^{k} \tilde{S}^{\left(j_{i}\right)}(p)}{\left[1+c\left(\gamma+\tilde{S}^{\prime}(p)\right)\right]^{k+1}} .
$$

Define the index set

$$
J(n, k)=\left\{j_{1}, \cdots, j_{k} \mid j_{1} \geq \cdots \geq j_{k} \geq 2, \sum_{i=1}^{k} j_{i}=n+k\right\} .
$$

Then claim for $n=2,3, \cdots$, that 


$$
\hat{\tilde{S}}^{(n)}(p)=\left[\sum_{k=1}^{n} \frac{\sum_{\left\{j_{i}\right\} \in J(n, k)} a_{n, k,\left\{j_{i}\right\}} \prod_{i=1}^{k} \tilde{S}^{\left(j_{i}\right)}(p)}{\left[1+c\left(\gamma+\tilde{S}^{\prime}(p)\right)\right]^{k+1}}\right](p-a)+\sum_{k=1}^{n-1} \frac{\sum_{\left\{j_{i}\right\} \in J(n-1, k)} b_{n, k,\left\{j_{i}\right\}} \prod_{i=1}^{k} \tilde{S}^{\left(j_{i}\right)}(p)}{\left[1+c\left(\gamma+\tilde{S}^{\prime}(p)\right)\right]^{k+1}} .
$$

Here the summation $\sum_{\left\{j_{i}\right\} \in J(n, k)} a_{n, k,\left\{j_{i}\right\}} \prod_{i=1}^{k} \tilde{S}^{\left(j_{i}\right)}(p)$ runs across all sets of indexes in $J(n, k)$ of the products $\prod_{i=1}^{k} \tilde{S}^{\left(j_{i}\right)}(p)$ times a constant associated with $n, k$ and the index set $\left\{j_{i}\right\}=\left\{j_{1}, \cdots, j_{k}\right\}$.

We can verify that this is true for $n=2 .^{34}$ Taking derivatives, we have

$$
\begin{aligned}
& \hat{\tilde{S}}^{(n+1)}(p)=\frac{d \hat{\tilde{S}}^{(n)}(p)}{d p} \\
& =\left[\sum_{k=1}^{n}\left[\frac{\sum_{\left\{j_{i}\right\} \in J(n, k)} a_{n, k,\left\{j_{i}\right\}} \sum_{l=1}^{k} \prod_{i=1}^{k} \tilde{S}^{\left(j_{i}+1_{(i l l)}\right)}(p)}{\left[1+c\left(\gamma+\tilde{S}^{\prime}(p)\right)\right]^{k+1}}+\frac{\left(-(k+1) c \tilde{S}^{(2)}(p)\right) \sum_{\left\{j_{i}\right\} \in J(n, k)} a_{n, k,\left\{j_{i}\right\}} \prod_{i=1}^{k} \tilde{S}^{\left(j_{i}\right)}(p)}{\left[1+c\left(\gamma+\tilde{S}^{\prime}(p)\right)\right]^{k+2}}\right](p-a)\right.
\end{aligned}
$$$$
+\sum_{k=1}^{n} \frac{\sum_{\left\{j_{i}\right\} \in J(n, k)} a_{n, k,\left\{j_{i}\right\}} \prod_{i=1}^{k} \tilde{S}^{\left(j_{i}\right)}(p)}{\left[1+c\left(\gamma+\tilde{S}^{\prime}(p)\right)\right]^{k+1}}
$$$$
+\sum_{k=1}^{n-1}\left[\frac{\sum_{\left\{j_{i}\right\} \in J(n-1, k)} b_{n, k,\left\{j_{i}\right\}} \sum_{l=1}^{k} \prod_{i=1}^{k} \tilde{S}^{\left(j_{i}+1_{(i=l)}\right)}(p)}{\left[1+c\left(\gamma+\tilde{S}^{\prime}(p)\right)\right]^{k+1}}+\frac{\left(-(k+1) c \tilde{S}^{(2)}(p)\right) \sum_{\left\{j_{i}\right\} \in J(n-1, k)} b_{n, k,\left\{j_{i}\right\}} \prod_{i=1}^{k} \tilde{S}^{\left(j_{i}\right)}(p)}{\left[1+c\left(\gamma+\tilde{S}^{\prime}(p)\right)\right]^{k+2}}\right]
$$$$
=\left[\sum_{k=1}^{n+1} \frac{\sum_{\left\{j_{i}\right\} \in J(n+1, k)} a_{n+1, k,\left\{j_{i}\right\}} \prod_{i=1}^{k} \tilde{S}^{\left(j_{i}\right)}(p)}{\left[1+c\left(\gamma+\tilde{S}^{\prime}(p)\right)\right]^{k+1}}\right](p-a)+\sum_{k=1}^{n} \frac{\sum_{\left\{j_{i}\right\} \in J(n, k)} b_{n+1, k,\left\{j_{i}\right\}} \prod_{i=1}^{k} \tilde{S}^{\left(j_{i}\right)}(p)}{\left[1+c\left(\gamma+\tilde{S}^{\prime}(p)\right)\right]^{k+1}} .
$$

Here,

\footnotetext{
34 Note that this is not true for $n=1$. The affine demand assumption implies a special transition from the first to the second derivative where we lose the demand slope in the numerator.
} 


$$
\begin{aligned}
& a_{n+1,1,\left\{j_{i}\right\}}=a_{n+1,1,\{n+2\}}=a_{n, 1,\{n+1\}}=1, \\
& a_{n, n+1,\{n+2\}}=0, \\
& \text { and for } k=2, \cdots, n+1, \\
& a_{n+1, k,\left\{j_{i}\right\}}=\sum_{l=1}^{k} a_{n, k,\left\{j_{i}+1_{(i=l)}\right\}}+(-(k) c) a_{n, k-1,\left\{j_{i}, j_{k}=2\right\}} .
\end{aligned}
$$

Here we intend that the summation $\sum_{l=1}^{k} a_{n, k,\left\{j_{i}+1_{(i=l)}\right\}}$ as the sum for all index sets in $J(n, k)$ with one element increased by +1 and sorted produces the corresponding index set in $J(n+1, k)$. And the selection $a_{n, k-1,\left\{j_{i}, j_{k}=2\right\}}$ means the index set from $J(n, k)$ with appended term $j_{k}=2$ produces the corresponding index set in $J(n+1, k)$.

$$
\begin{aligned}
& b_{n+1,1,\left\{j_{i}\right\}}=b_{n+1,1,\{n+1\}}=b_{n, 1,\{n\}}+a_{n, 1,\{n+1\}}=n+1, \\
& b_{n, n,\{2 n-1\}}=0, \\
& \text { and for } k=2, \cdots, n, \\
& b_{n+1, k,\left\{j_{i}\right\}}=a_{n, k,\left\{j_{i}\right\}}+\sum_{l=1}^{k} b_{n, k,\left\{j_{i}+1_{(i=l)}\right\}}+(-(k) c) b_{n, k-1,\left\{j_{i}, j_{k}=2\right\}} .
\end{aligned}
$$

We can show that the various coefficients $a_{n+1, k,\left\{j_{i}\right\}}, b_{n+1, k,\left\{j_{i}\right\}}$ are not zero (note that $k=$ odd terms are positive and $k=$ even terms are negative). Hence the claim for the general form of the derivative is established.

Let $\alpha_{i}=S^{(i)}\left(p_{0}\right)$. Then define the derivative of the best response to the approximation function as $\hat{\tilde{S}}^{(j)}(p)=g_{j}\left(\alpha_{1}, \alpha_{2}, \cdots, \alpha_{j+1}\right)$. We can address the stability of the fixed points for the Taylor approximation by computing the differential of $g$.

$$
g(\alpha, z)=\left(\begin{array}{c}
g_{1}\left(\alpha_{1}, \alpha_{2}\right) \\
g_{2}\left(\alpha_{1}, \alpha_{2}, \alpha_{3}\right) \\
g_{3}\left(\alpha_{1}, \alpha_{2}, \alpha_{3}, \alpha_{4}\right) \\
g_{4}\left(\alpha_{1}, \alpha_{2}, \alpha_{3}, \alpha_{4}, \alpha_{5}\right) \\
\vdots \\
g_{n}\left(\alpha_{1}, \alpha_{2}, \cdots, \alpha_{n-1}, \alpha_{n}, z\right)
\end{array}\right)
$$

The polynomial approximation of degree $n$ is the application of $g(\alpha, 0)$. Therefore, the differential matrix is 


$$
D g(\alpha, 0)=\left(\begin{array}{cccccc}
\frac{\partial g_{1}}{\partial \alpha_{1}} & \frac{\partial g_{1}}{\partial \alpha_{2}} & 0 & 0 & \ldots & 0 \\
\frac{\partial g_{2}}{\partial \alpha_{1}} & \frac{\partial g_{2}}{\partial \alpha_{2}} & \frac{\partial g_{2}}{\partial \alpha_{3}} & 0 & \ldots & 0 \\
\frac{\partial g_{3}}{\partial \alpha_{1}} & \frac{\partial g_{3}}{\partial \alpha_{2}} & \frac{\partial g_{3}}{\partial \alpha_{3}} & \frac{\partial g_{3}}{\partial \alpha_{4}} & \ldots & 0 \\
\vdots & \vdots & \vdots & \vdots & \vdots & \vdots \\
\frac{\partial g_{n-1}}{\partial \alpha_{1}} & \frac{\partial g_{n-1}}{\partial \alpha_{2}} & \frac{\partial g_{n-1}}{\partial \alpha_{3}} & \ldots & \frac{\partial g_{n-1}}{\partial \alpha_{n-1}} & \frac{\partial g_{n-1}}{\partial \alpha_{n}} \\
\frac{\partial g_{n}}{\partial \alpha_{1}} & \frac{\partial g_{n}}{\partial \alpha_{2}} & \frac{\partial g_{n}}{\partial \alpha_{3}} & \frac{\partial g_{n}}{\partial \alpha_{4}} & \ldots & \frac{\partial g_{n}}{\partial \alpha_{n}}
\end{array}\right)
$$

We can evaluate this at the affine equilibrium point.

$\operatorname{Dg}(\theta, 0,0, \cdots, 0)=\left(\begin{array}{cccccc}\frac{1}{[1+c(\gamma+\theta)]^{2}} & \frac{p-a}{[1+c(\gamma+\theta)]^{2}} & 0 & 0 & \cdots & 0 \\ 0 & \frac{2}{[1+c(\gamma+\theta)]^{2}} & \frac{p-a}{[1+c(\gamma+\theta)]^{2}} & 0 & \cdots & 0 \\ 0 & 0 & \frac{3}{[1+c(\gamma+\theta)]^{2}} & \frac{p-a}{[1+c(\gamma+\theta)]^{2}} & \cdots & 0 \\ \vdots & \vdots & \vdots & \vdots & \vdots & \vdots \\ 0 & 0 & 0 & \cdots & \frac{n-1}{[1+c(\gamma+\theta)]^{2}} & \frac{p}{[1+c(\gamma+\theta)]^{2}} \\ 0 & 0 & 0 & 0 & \cdots & \frac{n}{[1+c(\gamma+\theta)]^{2}}\end{array}\right)$.

This matrix is not diagonal but the upper triangular structure implies that the diagonal gives the distinct eigenvalues. Again we see that the largest eigenvalue is

$$
\frac{n}{[1+c(\gamma+\theta)]^{2}} \text {. }
$$

For $n$ greater than $n_{\text {affine }}^{c}=[1+c(\gamma+\theta)]^{2}$ we have the affine solution as locally unstable with respect to the polynomial approximation.

For any non-affine fixed points we can calculate the differential. For instance, in the case of $n=2$, at a polynomial approximation equilibrium we have 


$$
\begin{aligned}
& \operatorname{Dg}\left(S^{(1)}\left(p_{0}\right), S^{(2)}\left(p_{0}\right), S^{(3)}\left(p_{0}\right)\right) \\
& =\left(\begin{array}{cc}
\frac{2 c S^{(2)}\left(p_{0}\right)\left(p_{0}-a\right)}{\left[1+c\left(\gamma+S^{(1)}\left(p_{0}\right)\right)\right]} & \frac{p_{0}-a}{\left[1+c\left(\gamma+S^{(1)}\left(p_{0}\right)\right)\right]^{2}} \\
\left(\frac{-2 c S^{(2)}\left(p_{0}\right)}{\left.\left[1+c+S^{(1)}\left(p_{0}\right)\right)\right]^{2}}\right)\left(1-\frac{c S^{(2)}\left(p_{0}\right)\left(p_{0}-a\right)}{\left[1+c\left(\gamma+S^{(1)}\left(p_{0}\right)\right)\right]^{3}}\right) & \frac{4 c S^{(2)}\left(p_{0}\right)\left(p_{0}-a\right)}{\left[1+c\left(\gamma+S^{(1)}\left(p_{0}\right)\right)\right]} \\
{\left[1+c\left(\gamma+S^{(1)}\left(p_{0}\right)\right)\right]}
\end{array}\right) .
\end{aligned}
$$

In the case of $n=3$, at a polynomial approximation equilibrium we have

$$
\begin{aligned}
& D g\left(S^{(1)}\left(p_{0}\right), S^{(2)}\left(p_{0}\right), S^{(3)}\left(p_{0}\right), S^{(4)}\left(p_{0}\right)\right)= \\
& \left(\begin{array}{ccc}
\frac{1-\frac{2 c S^{(2)}\left(p_{0}\right)\left(p_{0}-a\right)}{\left[1+c\left(\gamma+S^{(1)}\left(p_{0}\right)\right)\right]}}{\left[1+c\left(\gamma+S^{(1)}\left(p_{0}\right)\right)\right]^{2}} & \frac{p_{0}-a}{\left[1+c\left(\gamma+S^{(1)}\left(p_{0}\right)\right)\right]^{2}} & \\
\frac{\partial g_{2}}{\partial \alpha_{1}} & \frac{4 c S^{(2)}\left(p_{0}\right)\left(p_{0}-a\right)}{\left[1+c\left(\gamma+S^{(1)}\left(p_{0}\right)\right)\right]} & \frac{p_{0}-a}{\left[1+c\left(\gamma+S^{(1)}\left(p_{0}\right)\right)\right]^{2}} \\
& \frac{\partial g_{3}}{\left[1+c\left(\gamma+S^{(1)}\left(p_{0}\right)\right)\right]^{2}} \\
\frac{\partial g_{3}}{\partial \alpha_{1}} & \frac{3-\frac{6 c S^{(2)}\left(p_{0}\right)\left(p_{0}-a\right)}{\left[1+c\left(\gamma+S^{(1)}\left(p_{0}\right)\right)\right]}}{\left[1+c\left(\gamma+S^{(1)}\left(p_{0}\right)\right)\right]^{2}}
\end{array}\right) \\
& \frac{\partial g_{2}}{\partial \alpha_{1}}=\left(\frac{-2 c S^{(2)}\left(p_{0}\right)}{\left[1+c\left(\gamma+S^{(1)}\left(p_{0}\right)\right)\right]}\right)\left(1-\frac{c\left[S^{(2)}\left(p_{0}\right)\right]\left(p_{0}-a\right)}{\left[1+c\left(\gamma+S^{(1)}\left(p_{0}\right)\right)\right]^{3}}\right), \\
& \frac{\partial g_{3}}{\partial \alpha_{1}}=\left(\frac{-2 c}{\left[1+c\left(\gamma+S^{(1)}\left(p_{0}\right)\right)\right]}\right)\left(\begin{array}{l}
S^{(3)}\left(p_{0}\right) \\
+\left(-\frac{(2+2 \cdot 2) c S^{(3)}\left(p_{0}\right) S^{(2)}\left(p_{0}\right)}{\left[1+c\left(\gamma+S^{(1)}\left(p_{0}\right)\right)\right]^{3}}+\frac{2 \cdot 2 \cdot 3 c^{2}\left[S^{(2)}\left(p_{0}\right)\right]^{3}}{\left[1+c\left(\gamma+S^{(1)}\left(p_{0}\right)\right)\right]^{4}}\right)\left(p_{0}-a\right) \\
-\frac{(2+2 \cdot 2) c\left[S^{(2)}\left(p_{0}\right)\right]^{2}}{\left[1+c\left(\gamma+S^{(1)}\left(p_{0}\right)\right)\right]^{3}}
\end{array}\right), \\
& \frac{\partial g_{3}}{\partial \alpha_{2}}=\left[\begin{array}{c}
-\frac{(2+2 \cdot 2) c S^{(3)}\left(p_{0}\right)}{\left[1+c\left(\gamma+S^{(1)}\left(p_{0}\right)\right)\right]^{3}} \\
+\frac{3 \cdot 2 \cdot 3 c^{2}\left[S^{(2)}\left(p_{0}\right)\right]^{2}}{\left[1+c\left(\gamma+S^{(1)}\left(p_{0}\right)\right)\right]^{4}}
\end{array}\right](p-a)-\frac{2(2+2 \cdot 2) c\left[S^{(2)}\left(p_{0}\right)\right]}{\left[1+c\left(\gamma+S^{(1)}\left(p_{0}\right)\right)\right]^{3}} .
\end{aligned}
$$




\section{Exact SFE Model}

Although we do not have a closed form for the exact unconstrained nonlinear SFEs, as shown in Klemperer and Meyer we can compute the trajectory of an arbitrary SFE using the autonomous system: ${ }^{35}$

$$
\begin{aligned}
& \widehat{S}^{\prime}(t)=\widehat{S}+\gamma(\hat{p}-a-c \widehat{S}), \\
& \hat{p}^{\prime}(t)=\hat{p}-a-c \widehat{S} .
\end{aligned}
$$

This system gives rise to the solutions:

$$
\begin{aligned}
& \hat{S}(t)=A_{1} e^{\lambda_{1} t} v_{1}+A_{2} e^{\lambda_{2} t} v_{2}, \\
& \hat{p}(t)=a+A_{1} e^{\lambda_{1} t}+A_{2} e^{\lambda_{2} t}, \\
& \lambda_{1}>1,0<\lambda_{2}<1 .
\end{aligned}
$$

Here the parameters are determined by the eigenvalues and eigenvectors for the autonomous system.

$$
\begin{aligned}
& \lambda_{1}=\left((2+\gamma c)+\left(\gamma^{2} c^{2}+4 \gamma c\right)^{1 / 2}\right) / 2, \\
& \lambda_{2}=\left((2+\gamma c)-\left(\gamma^{2} c^{2}+4 \gamma c\right)^{1 / 2}\right) / 2, \\
& v_{i}=\frac{1-\lambda_{i}}{c} .
\end{aligned}
$$

The parameters $A_{1}$ and $A_{2}$ are arbitrary and any $A_{2} \neq 0$ admits the same SFE with a proper choice of $A_{1}$. Then

$$
\begin{aligned}
& \hat{S}^{\prime}(t)=\lambda_{1} A_{1} e^{\lambda_{1} t} v_{1}+\lambda_{2} A_{2} e^{\lambda_{2} t} v_{2}, \\
& \hat{p}^{\prime}(t)=\lambda_{1} A_{1} e^{\lambda_{1} t}+\lambda_{2} A_{2} e^{\lambda_{2} t} .
\end{aligned}
$$

Now, with $t(p)$ as the inverse mapping of $\hat{p}(t)$,

$$
\begin{aligned}
& S(p)=\hat{S}(t(p)) . \\
& S^{\prime}(p)=\hat{S}^{\prime}(t(p)) t^{\prime}(p) .
\end{aligned}
$$

By the usual argument,

$$
\begin{aligned}
& \hat{p}(t(p))=p, \\
& \hat{p}^{\prime}(t(p)) t^{\prime}(p)=1 .
\end{aligned}
$$

Therefore,

$$
t^{\prime}(p)=\frac{1}{\hat{p}^{\prime}(t(p))}
$$


Hence,

$$
\begin{aligned}
S^{\prime}(p) & =\widehat{S}^{\prime}(t(p)) t^{\prime}(p) \\
& =\frac{\left[\lambda_{1} A_{1} e^{\lambda_{1} t} v_{1}+\lambda_{2} A_{2} e^{\lambda_{2} t} v_{2}\right]}{\left[\lambda_{1} A_{1} e^{\lambda_{1} t}+\lambda_{2} A_{2} e^{\lambda_{2} t}\right]} .
\end{aligned}
$$

Given the benchmark demand curve, we can solve numerically for the $A_{1}$ and $t_{\max }$ that achieve any particular slope at the intersection with (half) the demand curve.

\title{
-end-
}

\begin{abstract}
${ }^{i}$ Ross Baldick is with the Department of Electrical and Computer Engineering, The University of Texas at Austin. The author is or has been a consultant to Tenneco Power Generation Company, The California Energy Commission, Bracewell and Patterson LLP, The South Texas Electric Cooperative, Reliant Energy, Dynegy Corporation, Colonial Pipeline, The Federal Energy Regulatory Commission, Texas Competitive Power Advocates, Boston Pacific.

${ }^{i i}$ William W. Hogan is the Lucius N. Littauer Professor of Public Policy and Administration, John F. Kennedy School of Government, Harvard University and a Director of LECG, LLC. This paper draws on work for the Harvard Electricity Policy Group and the Harvard-Japan Project on Energy and the Environment. The author is or has been a consultant on electric market reform and transmission issues for Allegheny Electric Global Market, American Electric Power, American National Power, Australian Gas Light Company, Avista Energy, Brazil Power Exchange Administrator (ASMAE), British National Grid Company, California Independent Energy Producers Association, Calpine Corporation, Central Maine Power Company, Comision Reguladora De Energia (CRE, Mexico), Commonwealth Edison Company, Conectiv, Constellation Power Source, Coral Power, Detroit Edison Company, Duquesne Light Company, Dynegy, Edison Electric Institute, Edison Mission Energy, Electricity Corporation of New Zealand, Electric Power Supply Association, El Paso Electric, GPU Inc. (and the Supporting Companies of PJM), GPU PowerNet Pty Ltd., GWF Energy, Independent Energy Producers Assn, ISO New England, Maine Public Advocate, Maine Public Utilities Commission, Midwest ISO, Mirant Corporation, Morgan Stanley Capital Group, National Independent Energy Producers, New England Power Company, New York Independent System Operator, New York Power Pool, New York Utilities Collaborative, Niagara Mohawk Corporation, NRG Energy, Inc., Ontario IMO, Pepco, Pinpoint Power, PJM Office of Interconnection, PP\&L, Public Service Electric \& Gas Company, Reliant Energy, Rhode Island Public Utilities Commission, San Diego Gas \& Electric Corporation, Sempra Energy Resources, SoCalGas, SPP, Texas Utilities Co, TransÉnergie, Transpower of New Zealand, Westbrook Power, Williams Energy Group, and Wisconsin Electric Power Company. (Related papers can be found on the web the web at www.whogan.com).
\end{abstract}

The authors received helpful comments from Steven Anderson, Scott Harvey, Richard O’Neill, and Aleksandr Rudkevich. The views presented here are not necessarily attributable to any of those mentioned, and any remaining errors are solely the responsibility of the authors. 